\title{
An Experimental Study of the Ventral Striatum of the Golden Hamster. I. Neuronal Connections of the Nucleus Accumbens
}

\author{
RICHARD NEWMAN AND SARAH SCHILLING WINANS \\ Department of Anatomy, The University of Michigan Medical School, Ann Arbor, \\ Michigan 48109
}

\begin{abstract}
As part of an experimental study of the ventral striatum, the horseradish peroxidase (HRP) method was used to examine the afferent and efferent neuronal connections of the nucleus accumbens. Following iontophoretic applications or hydraulic injections of HRP in nucleus accumbens, cells labeled by retrograde transport of HRP were observed in the ipsilateral telencephalon in the posterior agranular insular, perirhinal, entorhinal, and primary olfactory cortices, in the subiculum and hippocampal field CA1, and in the anterior and posterior divisions of the basolateral amygdaloid nucleus. In the diencephalon, labeled neurons were present ipsilaterally in the central medial, paracentral and parafascicular intralaminar nuclei, and in the midline nuclei parataenialis, paraventricularis, and reuniens. Retrograde labeling was observed in the ipsilateral brainstem in cells of the ventral tegmental area and dorsal raphe. Many of these projections to nucleus accumbens were found to be topographically organized.

Anterograde transport of HRP from nucleus accumbens demonstrated ipsilateral terminal fields in the ventral pallidum and substantia nigra, pars reticulata.

The afferent projections to nucleus accumbens from the posterior insular and perirhinal neocortices, intralaminar thalamus, and the dopamine-containing ventral tegmental area are analogous to the connections of the caudatoputamen, as are the efferents from nucleus accumbens to the substantia nigra and ventral globus pallidus. These connections substantiate the classification of nucleus accumbens as a striatal structure and provide support for the recently proposed concept of the ventral striatum. Furthermore, the demonstration that a number of limbic system structures, including the amygdala, hippocampal formation, entorhinal cortex, and olfactory cortex are important sources of afferents to the nucleus accumbens, suggests that the ventral striatum may serve to integrate limbic information into the striatal system.
\end{abstract}

It has recently been suggested that the nucleus accumbens, olfactory tubercle, and substriatal gray-three apparently unrelated basal forebrain areas - are anatomically and functionally related striatal structures. Heimer and Wilson ('75) have applied the term "ventral striatum" to these areas collectively and have provided evidence that they project to a subcommissural portion of the globus pallidus, which these investigators have called the "ventral pallidum." Heimer and Wilson have drawn attention to the fact that while the neuronal connections of this ventral striato-pallidal system are in part analogous to those of the classical dorsal system, the known telencephalic afferents of the ventral striatum are largely from the hippocampus, primary olfactory cortex, and amygdala, and thus limbic in nature, in contrast to the neocortical input to the dorsal system. The ventral striatum, then, is one of the few areas in which the limbic and striatal systems interface directly.

The nucleus accumbens is the most prominent component of the ventral striatum. The functional significance of this nucleus has historically been a topic of considerable debate; accumbens has been variously included in the olfactory, limbic, and striatal systems because of its intermediate location between the caudatoputamen and septum, and because of the multitude of connections ascribed to it by 
early investigators. These reported connections include efferents from nucleus accumbens to the septum and caudate nucleus of the macaque (Lauer, '45), the substantia nigra in the tamandua and cat (Smith, '30; Rioch, '31), the globus pallidus and the entopeduncular nucleus of the tamandua (Smith, '30), the rat hypothalamus and amygdala (Gurdjian, '27, '28), and the dorsomedial thalamic nucleus of the cat (Guillery, '59). Afferents to nucleus accumbens have been described from the cat hippocampus (Fox, '43), the macaque olfactory tubercle (Lauer, '45), the amygdala of the rat (Knook, '66), and the parataenial thalamic nucleus in the rabbit and monkey (Cowan and Powell, '55; Powell and Cowan, '56).

The application of modern experimental techniques to this problem has provided information suggesting that nucleus accumbens is anatomically related to both the caudatoputamen and the limbic system. Swanson and Cowan ('75), using tritiated thymidine, have demonstrated a close ontogenetic relationship between nucleus accumbens and the caudatoputamen, while Heimer and Wilson ('75) have called attention to the histochemical and cytoarchitectural similarities of these two areas. Furthermore, autoradiographic experiments by a number of researchers have shown the efferent projections of nucleus accumbens to be largely to the globus pallidus and substantia nigra, and thus similar to the efferents of the caudatoputamen (Swanson and Cowan, '75; Conrad and Pfaff, 76; Domesick et al., 76). In addition to efferents typical of the striatum, however, projections from nucleus accumbens to limbic areas, including the preoptic area and hypothalamus, have been proposed (Conrad and Pfaff, '76; Williams et al., '77; Nauta et al., '78).

Although the afferents of nucleus accumbens have never been systematically studied with modern methods, this nucleus, like the caudatoputamen, is now known to receive afferent fibers from dopaminergic neurons of the ventral midbrain (Ungerstedt, '71; Björklund and Lindvall, '78; Fallon and Moore, '78), and projections to the nucleus accumbens have recently been reported from limbic structures as well, including the subiculum and amygdala (Swanson and Cowan, '77; Krettek and Price, '78a).

Pharmacological and electrophysiological studies also suggest that nucleus accumbens is related to both the striatum and the limbic system. Dill and Costa ('77) have found that injections of morphine into accumbens produce catalepsy, and Pijenburg et al. ('73) have shown that injections of ergometrine cause a substantial increase in motor activity, while Smith and Holland ('75) have produced alterations in lactation in rats by placing lesions in nucleus accumbens, and Koikegami et al. ('67) have shown electrical stimulation of this nucleus to induce ovulation in rabbits.

In summary, nucleus accumbens is apparently a striatal structure closely associated functionally and anatomically with the limbic system. The concept of the ventral striatum may provide a basis for understanding this relationship. In order to test the validity of this concept experimentally, and to further define the anatomy of the ventral striatum, the afferent and efferent neuronal connections of the nucleus accumbens and the olfactory tubercle, which together compose the majority of the ventral striatum, were examined using the method of axonal transport of the protein horseradish peroxidase (HRP). The results of HRP experiments in the nucleus accumbens are presented here; those pertaining to the olfactory tubercle will be described in a second paper in this series (Newman and Winans, this issue).

\section{METHODS}

Fifty male and female golden hamsters (Mesocricetus auratus) ranging in weight from 75 to $130 \mathrm{~g}$ received injections or iontophoretic applications of HRP into the nucleus accumbens or neighboring forebrain structures. The hamsters were anesthetized with sodium pentobarbital $(75 \mathrm{mg} / \mathrm{kg})$, placed in a Kopf stereotaxic instrument, and subjected to one of the two procedures described below.

\section{Iontophoresis}

Horseradish peroxidase (Miles Laboratories, Inc., Elkhart, Indiana) was applied iontophoretically in 20 hamsters. The HRP was prepared at a concentration of $500 \mu \mathrm{g} / \mu \mathrm{l}$ in 0.05 M Tris buffer ( $\mathrm{pH}$ 8.6) and delivered through a glass micropipette having a tip diameter of 25-40 $\mu \mathrm{m}$. Anodal DC pulses of $1.5-3.0 \mathrm{mi}-$ croamps (one per second; 500 msec duration) were applied with a Grass SD5 or S88 stimulator. Current was applied for 10-30 minutes and the pipette remained in place for $10 \mathrm{~min}$ utes after the current pulses were stopped before it was withdrawn from the brain. During this time, a constant cathodal direct current of $0.15-0.30$ microamps was passed to prevent diffusion of HRP out of the pipette. 
Eighteen to 48 hours after surgery, the animals were reanesthetized and perfused through the heart with $250 \mathrm{ml}$ of cacodylatebuffered saline, followed by $250 \mathrm{ml}$ of a fixative containing $3 \%$ glutaraldehyde, or $1 \%$ paraformaldehyde and $1.5 \%$ glutaraldehyde. The brains were removed from the skulls and washed overnight in cold $\left(4^{\circ} \mathrm{C}\right) 25 \%$ sucrose prepared in the cacodylate-buffered saline solution. The pia was then removed with a rubber eraser, the brains cut coronally in $\mathbf{4 0}$ $\mu \mathrm{m}$ sections on a freezing microtome, and the sections stored in a cold solution of $25 \%$ sucrose in ethylene glycol for up to 24 hours before every fifth section was reacted for HRP. Perfusion, pia removal, and storage of the sections followed the procedure described by Hardy and Heimer ('77), except that the brains were placed in sucrose solution after removal rather than in fixative, the cut sections were collected in sucrose-ethylene glycol rather than in sodium sulphate, and in a number of cases, the fixative was modified by eliminating the paraformaldehyde, as described above.

Sections of these brains were reacted for HRP using the benzidine dihydrochloride (BDHC) method of de Olmos ('77), or the tetramethylbenzidine (TMB) procedure of Hardy and Heimer ('77). Reacted sections were mounted, air dried, and the TMB reacted sections were counterstained with $0.1 \%$ safranin$O$. Sections adjacent to those reacted for HRP using BDHC were mounted and stained with cresyl violet. All sections were quickly dehydrated and coverslipped. The slides were studied microscopically, using both brightfield and darkfield illumination.

\section{Pressure injections}

Hydraulic injections were made in 30 hamsters with a Hamilton syringe and 26-gauge needle using HRP (Sigma II or VI, or Miles) which had been prepared in $0.9 \%$ saline at concentrations of $100-500 \mu \mathrm{g} / \mu \mathrm{l}$. The HRP $(0.025-0.5 \mu \mathrm{l})$ was delivered over a period of ten minutes with the aid of a microinjector unit attached to the stereotaxic apparatus. The needle was left in the brain for ten minutes following the injection, then removed slowly.

The hamsters were allowed to survive 18-48 hours after surgery. They were then reanesthetized and perfused. In 14 animals, perfusion and subsequent processing to the point of reacting for HRP followed the procedure described above for the iontophoresis experiments. In 16 cases, the perfusion con- sisted of 250 cc of $0.1 \mathrm{~N}$ cacodylate-sucrose solution ( $\mathrm{pH} 7.2$; $3 \%$ sucrose), followed by 250 cc of fixative containing $5 \%$ glutaraldehyde in $0.1 \mathrm{~N}$ cacodylate buffer. The brains were removed from the skulls following perfusion, postfixed for four hours in cold $\left(4^{\circ} \mathrm{C}\right) 25 \%$ sucrose solution prepared in the perfusion fixative, then washed for 10-15 hours in cold $25 \%$ sucrose prepared in $0.1 \mathrm{~N}$ cacodylate buffer. Coronal sections were cut on a freezing microtome and stored in buffered sucrose for up to 24 hours before every fifth section was reacted for HRP.

Three different histochemical procedures were used in these experiments. In three cases, the diaminobenzidine (DAB) method of Graham and Karnovsky ('66) was followed, with

\section{Abbreviations}

AC anterior commissure

Acc nucleus accumbens

Bla basolateral amygdaloid nucleus, anterior division

Blp basolateral amygdaloid nucleus, posterior division

C1 anterior cortical amygdaloid nucleus

C2 posterolateral cortical amygdaloid nucleus

C3 posteromedial cortical amygdaloid nucleus

CA1 hippocampal field CA1

Ce central amygdaloid nucleus

CM central medial thalamic nucleus

CP caudatoputamen

d anterior olfactory nucleus, pars dorsalis

DR dorsal raphe

FR fasiculus retroflexus

GP globus pallidus

H2 Forel's field H2

IPN interpeduncular nucleus

1 anterior olfactory nucleus, pars lateralis

L lateral amygdaloid nucleus

LOT lateral olfactory tract

m anterior olfactory nucleus, pars medialis

M medial amygdaloid nucleus

NLOT nucleus of the lateral olfactory tract

OTu olfactory tubercle

p anterior olfactory nucleus, pars posterior

PC paracentral thalamic nucleus

PF parafasicular thalamic nucleus

PIC posterior agranular insular cortex

POC primary olfactory cortex

PR perirhinal cortex

PT parataenial thalamic nucleus

PV paraventricular thalamic nucleus

RE thalamic nucleus reuniens

RS rhinal sulcus

S subiculum

SC suprachiasmatic nucleus

Sept septum

So supraoptic nucleus

TR

tt

$\mathrm{v}$

VMH

VP

VTA

$28 \mathrm{~L}$

28L' entorhinal cortical area $T R$

taenia tecta

anterior olfactory nucleus, pars ventralis

ventromedial hypothalamic nucleus

ventral pallidum

ventral tegmental area

entorhinal cortical area $28 \mathrm{~L}$

entorhinal cortical area $28 \mathrm{~L}^{\prime}$ 

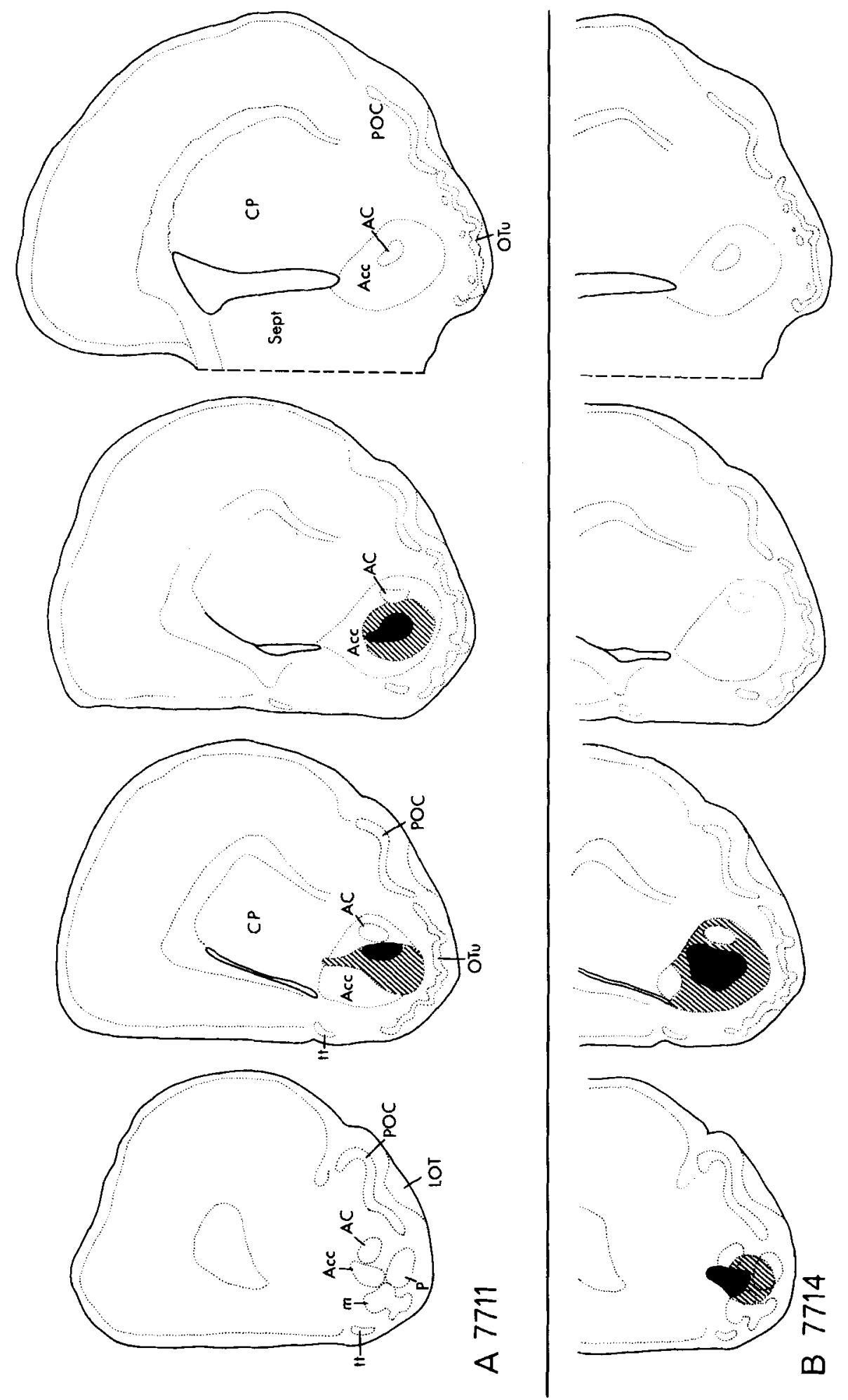
CONNECTIONS OF THE NUCLEUS ACCUMBENS

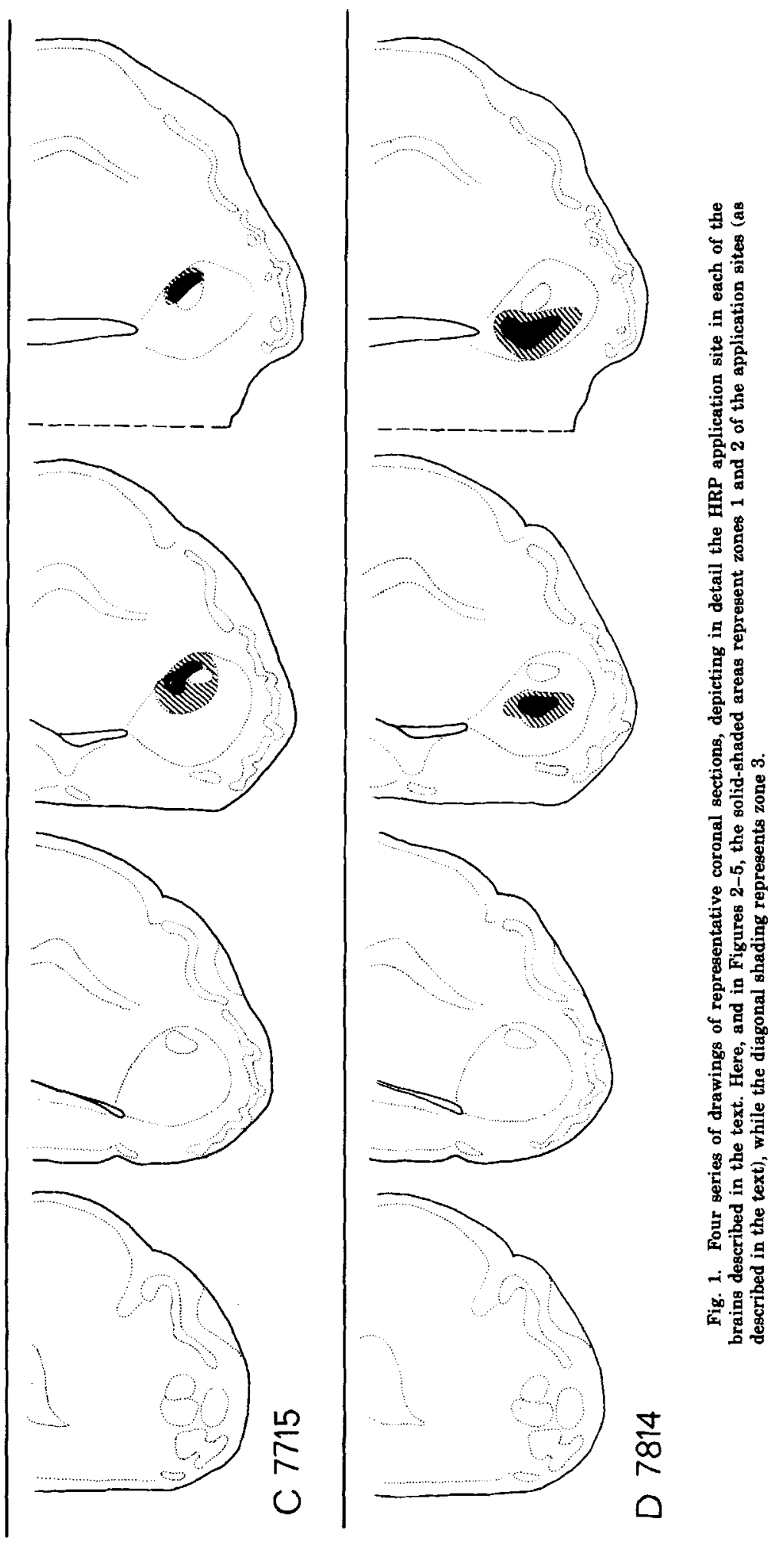



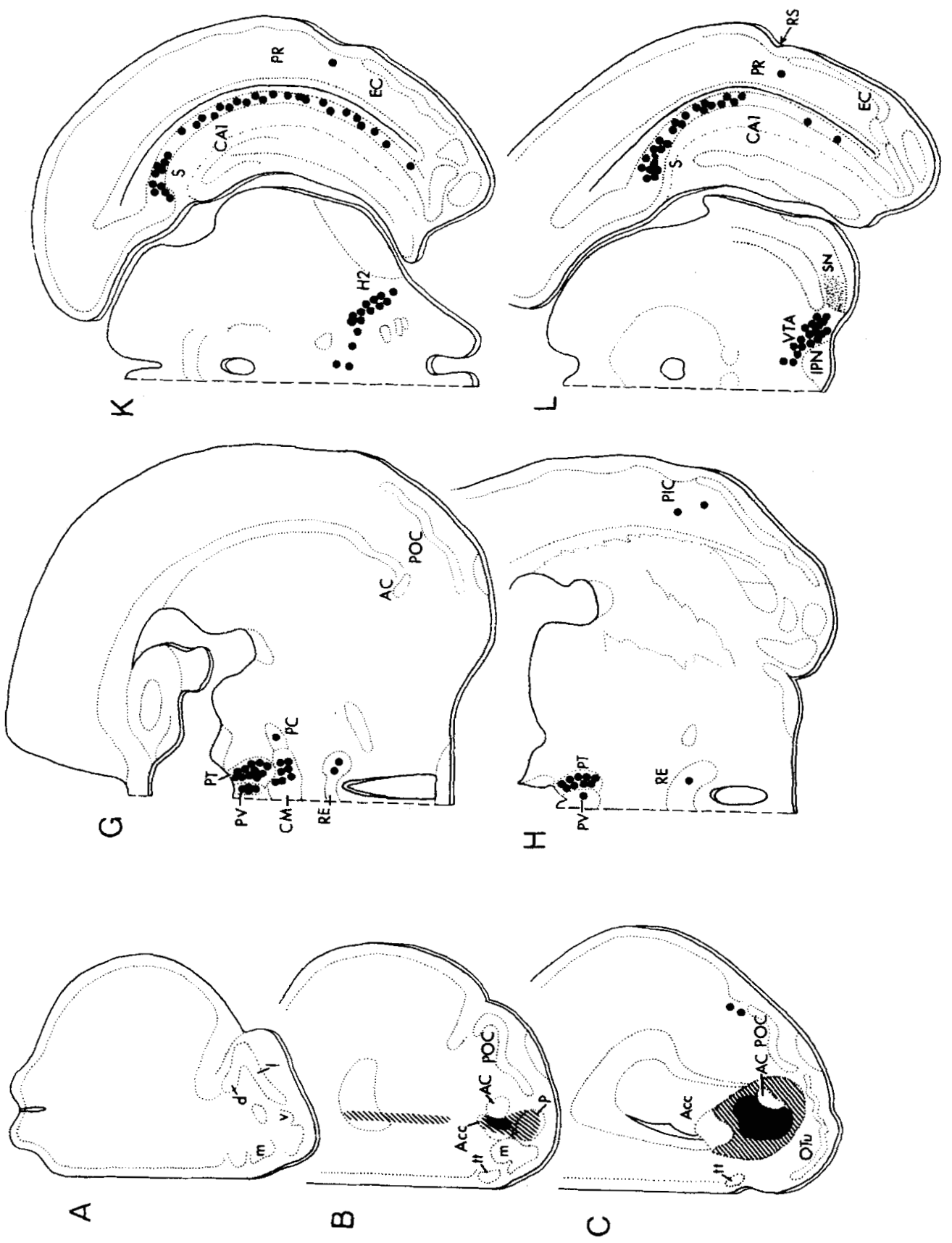


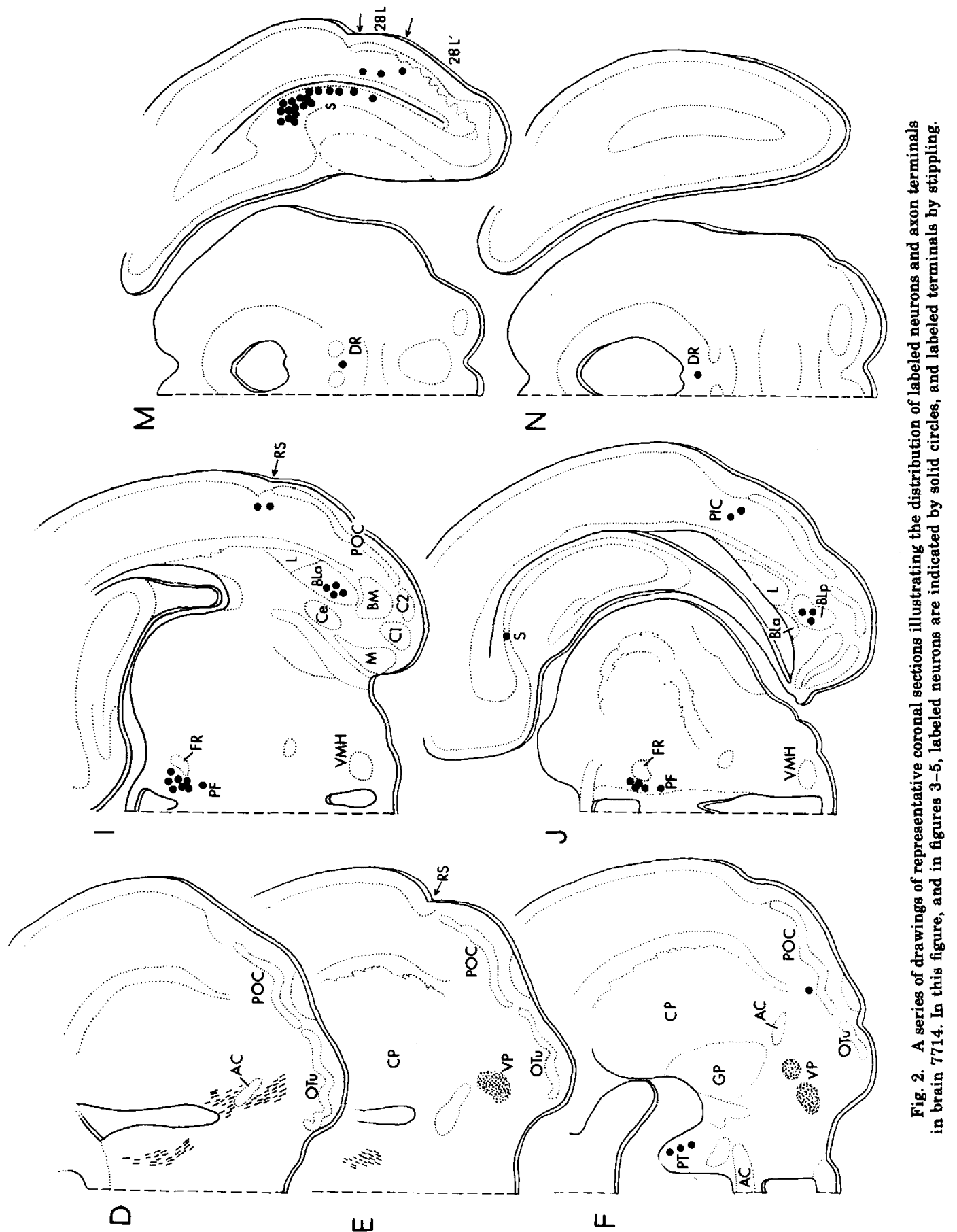



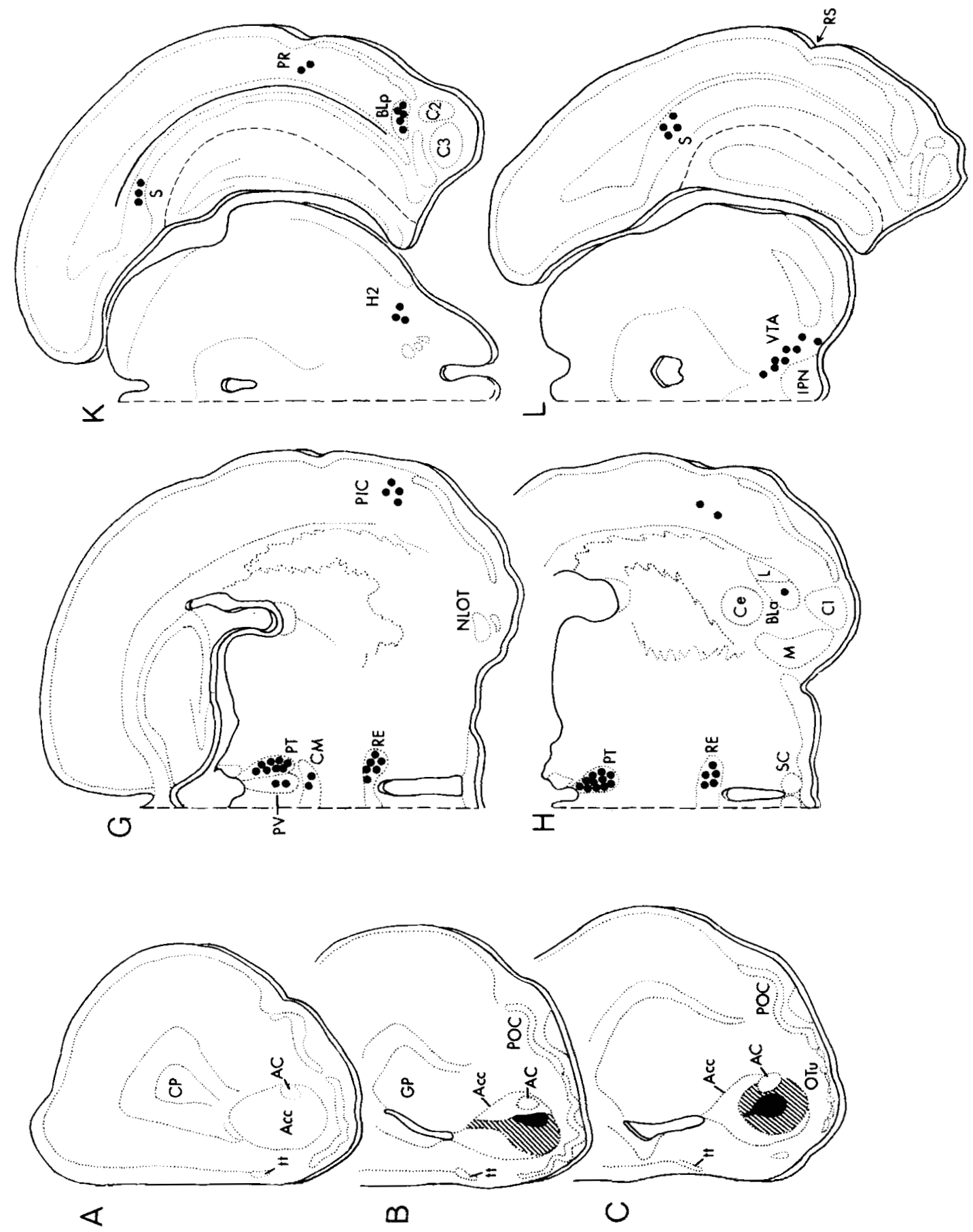

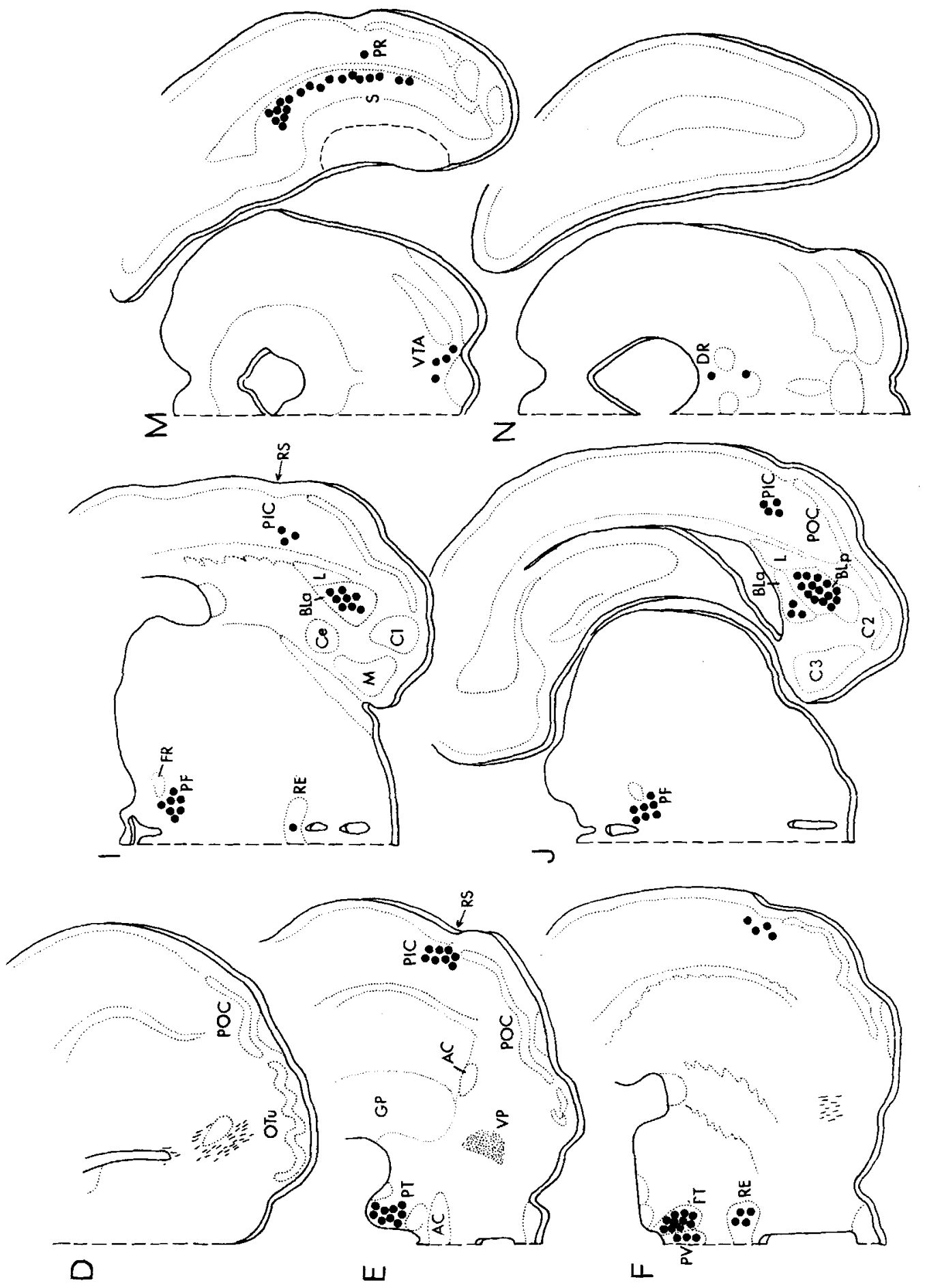
the exception that $0.1 \mathrm{M}$ sodium cacodylate (pH 7.2) was substituted for Tris buffer. Sections of 13 other brains were processed with the $\mathrm{DAB}$ procedure described by Colman et al. ('76), and an additional 14 brains were reacted for HRP using Colman and co-workers' o-dianisidine technique (76). Reacted sections and sections adjacent to those treated with DAB were mounted on gelatin-coated slides and air dried. Both the unreacted sections and those developed with o-dianisidine were stained with cresyl violet. All sections were then rapidly dehydrated and coverslipped prior to darkfield and brightfield microscopical examination.

\section{RESULTS}

\section{Technical considerations}

Factors influencing the size and characteristics of an HRP application site; identification of the application sites

The size of an HRP application site depended on the amount and concentration of the HRP introduced and on the method of application. Very small application sites restricted to nucleus accumbens were more reliably produced when HRP was applied iontophoretically than when injected under pressure. In addition, much less HRP was deposited along the pipette tract than the needle tract. The apparent size of an application site also depended on the histochemical technique used to visualize the HRP, as reported by de Olmos ('77) and Hardy and Heimer ('77).

At the end of the 18 to 48 hour survival periods, three regions were observed within the HRP application sites (Fig. 10): 1) a central area in which the cells were densely filled with granules of HRP reaction product, and in which a large amount of dense, finely granular reaction product was present in the extracellular space; 2) a middle region surrounding the central zone in which cellular labeling was similar to that described above, but in which only a moderate amount of reaction product was present in the extracellular space; and 3) a peripheral area in which small amounts of granular reaction product were present in cells only.

In this study, it was assumed that transport of HRP could occur only from regions (1) and (2), since the HRP concentration in region (3) was very low. Therefore, an application site was described as restricted to a given cytoarchitectonic area only if the central and middle regions did not extend beyond the boundaries of that area. In a number of cases all three regions were within the target area.

Identification of labeled cell bodies and terminal fields

Neurons were said to be labeled by retrograde transport of HRP if they were outside the application site and contained discrete granular HRP reaction product in the perinuclear cytoplasm.

Terminal fields labeled by orthograde transport of HRP appeared as clouds of coarsely granular reaction product distributed through the neuropil in a cytoarchitecturally defined area. They were seen in the same locations regardless of survival time in animals having HRP applications in the same areas of the brain, and at considerable distances from the application site, indicating that HRP reached these areas by axonal transport and not by diffusion within damaged axons.

\section{Experimental results}

Analysis of the experiments performed in the present study demonstrates that many of the connections of nucleus accumbens are topographically organized. In order to demonstrate these topographical relationships, four HRP applications varying in location within nucleus accumbens will be described. The application sites are shown in detail in Figure 1.

\section{Rostral nucleus accumbens}

Brain 7714 (Fig. 2) is an example of a large iontophoretic application of HRP involving the rostral pole of nucleus accumbens as well as the caudal-most portion of the anterior olfactory nucleus, pars posterior. The center of the iontophoresis site was medial to the anterior limb of the anterior commissure, at a level rostral to the genu of the corpus callosum (Figs. 1B; 2B,C). The survival time in this experiment was 36 hours.

In the ipsilateral telencephalon, a sparse band of labeled neurons was present along the caudal three-fourths of the rhinal sulcus. Peroxidase-containing pyramidal cells were observed in the posterior agranular insular cortex (terminology of Krettek and Price, '77a) from the level of the application site to the level of the entorhinal cortex (Fig. 2C, $\mathrm{H}-J$ ), and in the perirhinal cortex (Fig. 2K-M), caudal to the posterior insular cortex. Lamina III of the primary olfactory cortex (Fig. $2 \mathrm{~F}, \mathrm{H}$ ) and the deep layers of entorhinal cortical area 28L (of Haug, '76) also contained occasional labeled pyramidal cells (Fig. 2M). 
Other ipsilaterally labeled telencephalic areas included the caudal portion of the anterior division of the basolateral amygdaloid nucleus (terminology of Krettek and Price, 78b) (Fig. 2I) and the rostral portion of the poterior division of this nucleus (Fig. 2J). Peroxidase-containing pyramidal cells were also present in the stratum pyramidale of field CA1 of the intermediate hippocampus (Figs. $2 \mathrm{~K}, \mathrm{~L} ; 9)$ and in the dorsal and intermediate subiculum (Figs. 2J-M; 9), from the level of the posterior commissure caudally.

In the diencephalon, labeled cells were located ipsilaterally in the central medial, paracentral, and parafascicular intralaminar nuclei (Figs. 2G,I,J; 7). Labeled neurons within nucleus parafascicularis were medial and ventral to the fasciculus retroflexus, and located in the most rostral portion of the nucleus only. Labeled midline nuclei included parataenialis (Figs. 2F-H; 7), which contained many heavily labeled cells throughout its rostrocaudal extent, paraventricularis (Figs. 2G,H; 7), which was also well labeled, and reuniens, in which a number of large, densely labeled multipolar cells were observed (Fig. 2G,H).

Labeling was observed in the ipsilateral brainstem in the medial raphe, in the caudal mesencephalic portion of the dorsal raphe (Fig. 2M,N), and in the ventral tegmental area, which contained many large, heavily labeled neurons spreading medially over the interpeduncular nucleus to the midline (Figs. $2 \mathrm{~L} ; 8)$. Cells similar in appearance to those in the ventral tegmental area, and continuous with them, extended rostrally into the subthalamus in the region medial to Forel's field H2 (Fig. 2K).

A dense terminal field was labeled in the ipsilateral ventral pallidum of this brain. Small bundles of HRP-containing fibers within the dorsal portion of the medial forebrain bundle passed caudally from the iontophoresis site to the level of the body of the anterior commissure where many of them terminated within the ventral pallidum (Fig. $2 E, F)$. The remainder of these fibers continued past the ventral pallidum, but the number of labeled axons was much reduced caudal to this terminal field. A faint terminal field was also present ipsilaterally in the medial substantia nigra, pars reticulata (Fig. 2L).

\section{Intermediate nucleus accumbens}

The iontophoretic application of HRP in brain 7711 (Fig. 3) was centered more caudally in nucleus accumbens than that in experiment
7714 (Fig. 2) and was completely restricted to this nucleus (Figs. 1A; 3B,C). The survival time in this case was 18 hours.

With certain exceptions, the distribution of labeled cells in this experiment was similar to that observed after the HRP application in the rostral pole of nucleus accumbens in brain 7714. Differences included the absence of labeling in this brain in the primary olfactory cortex, entorhinal cortex, paracentral thalamic nucleus, hippocampal field CA1, and medial raphe. Also, fewer cells were labeled in the ventral tegmental area (Fig. 3L,M). In contrast to experiment 7714 in which the insular area and amygdala were only lightly labeled, the posterior insular cortex was heavily labeled in this brain (Fig. 3E-J), and the basolateral amygdaloid nucleus contained numerous labeled cells, especially the posterior division, which was labeled throughout its rostrocaudal extent (Fig. 3I-K).

As in experiment 7714 , a prominent terminal field was present in the ventral pallidum (Figs. 3E; 11). A terminal field was not present in the substantia nigra, however.

The areas mentioned above as being labeled in brain 7714 , but not in this brain (7711), may have been labeled only in brain 7714 because of the more rostral application site in that experiment. Alternatively, these areas may have been labeled by transport of HRP from the anterior olfactory nucleus, the caudal-most part of which was involved in the application site in experiment 7714 . The results of control experiments in which HRP was deposited in the anterior olfactory nucleus, and of additional experiments in which applications of HRP were made in nucleus accumbens (two of these are described below) demonstrate that the paracentral nucleus, primary olfactory cortex, hippocampal field CA1, and entorhinal cortex are indeed efferent to accumbens, and that nucleus accumbens does project to the substantia nigra. The medial raphe was labeled in brain 7714 by transport of HRP from the anterior olfactory nucleus.

\section{Caudolateral nucleus accumbens}

The application site in experiment $\mathbf{7 7 1 5}$ (Fig. 4) was centered in the lateral half of nucleus accumbens (Figs. $1 \mathrm{C} ; 4 \mathrm{~B}, \mathrm{C}$ ) at a level caudal to that of either of the two experiments described above (brains 7711 and 7714). The survival time in this experiment was 36 hours.

With the exception of hippocampal field CA1, areas labeled by retrograde transport of HRP from more rostral areas of nucleus ac- 
178

R. NEWMAN AND S.S. WINANS
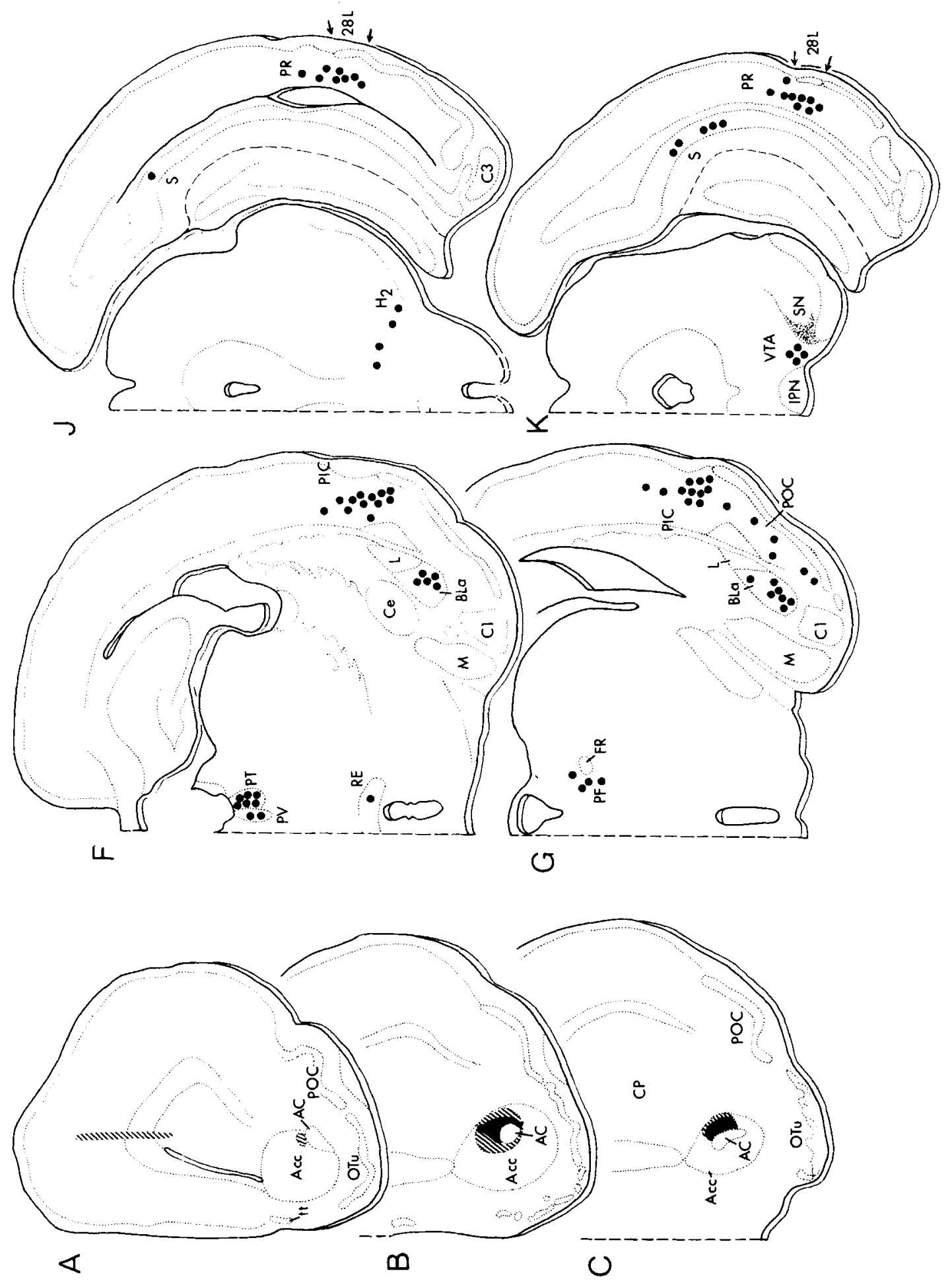

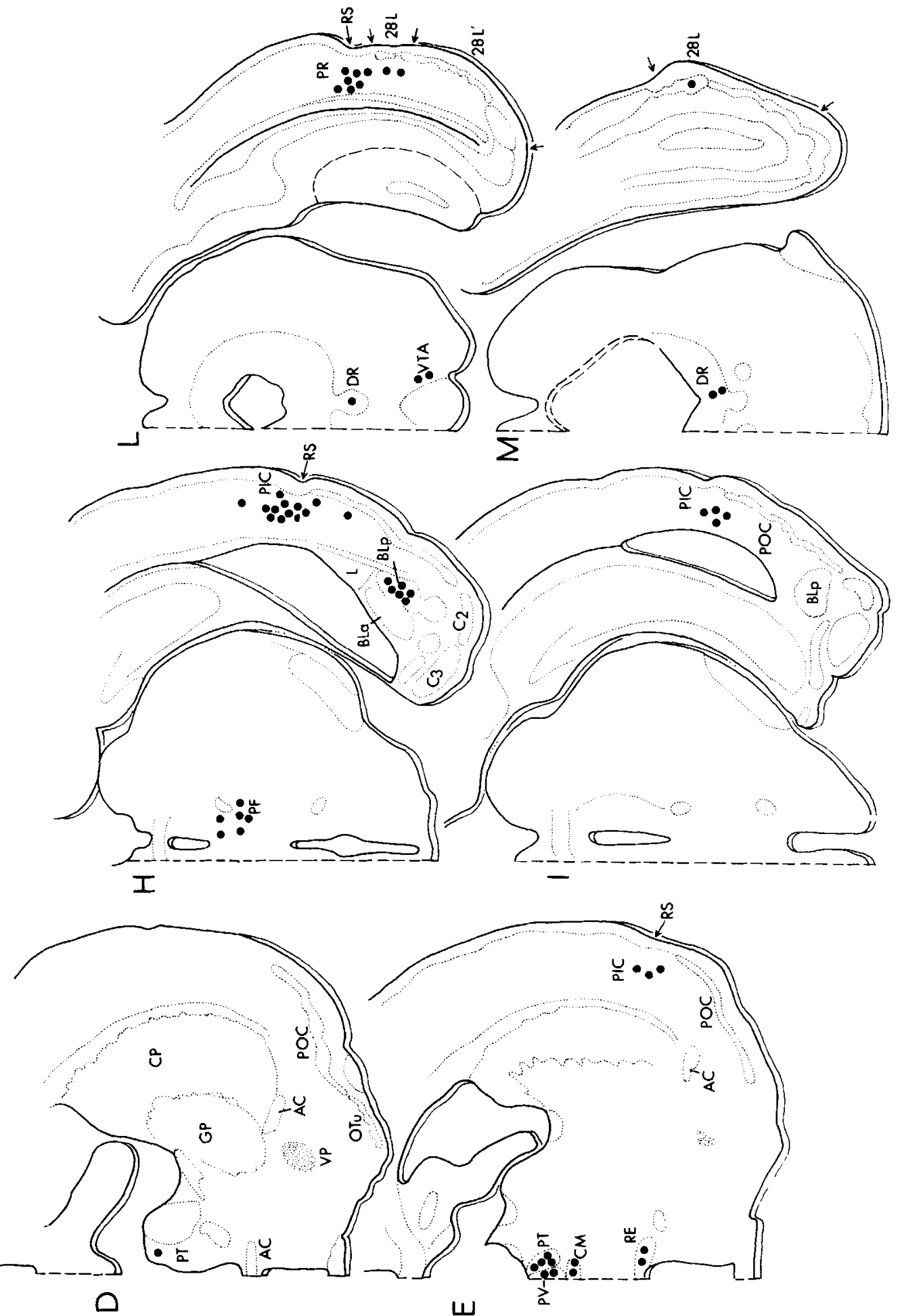

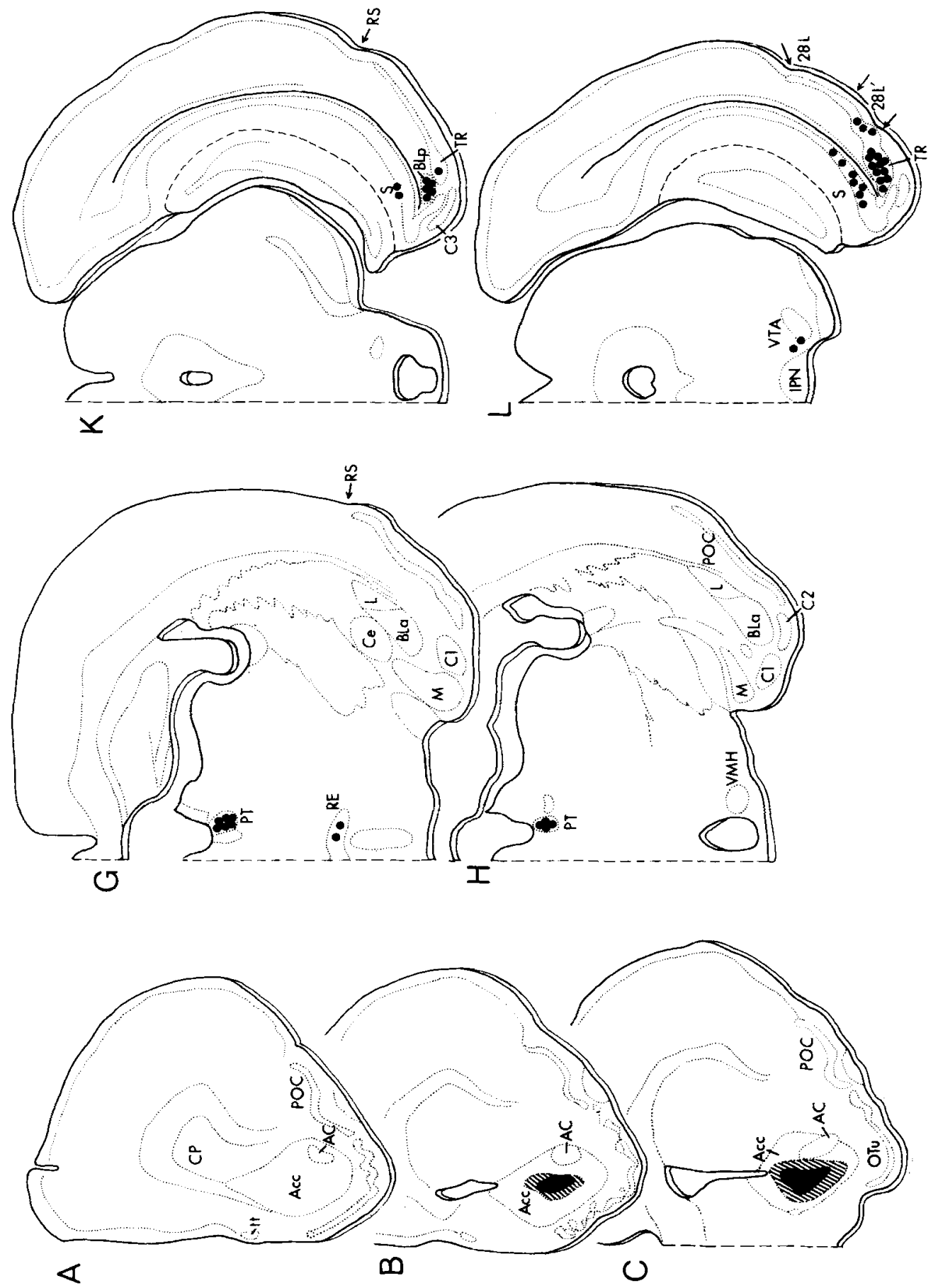


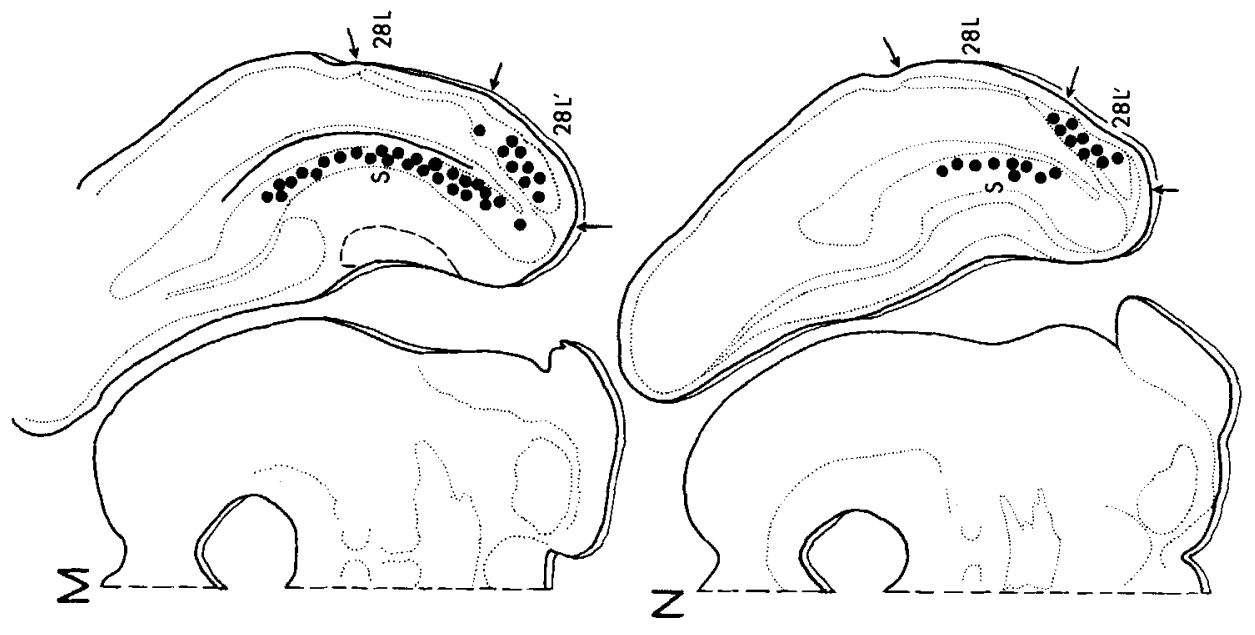

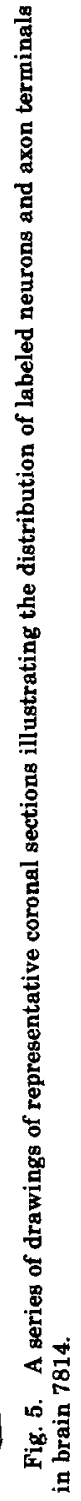

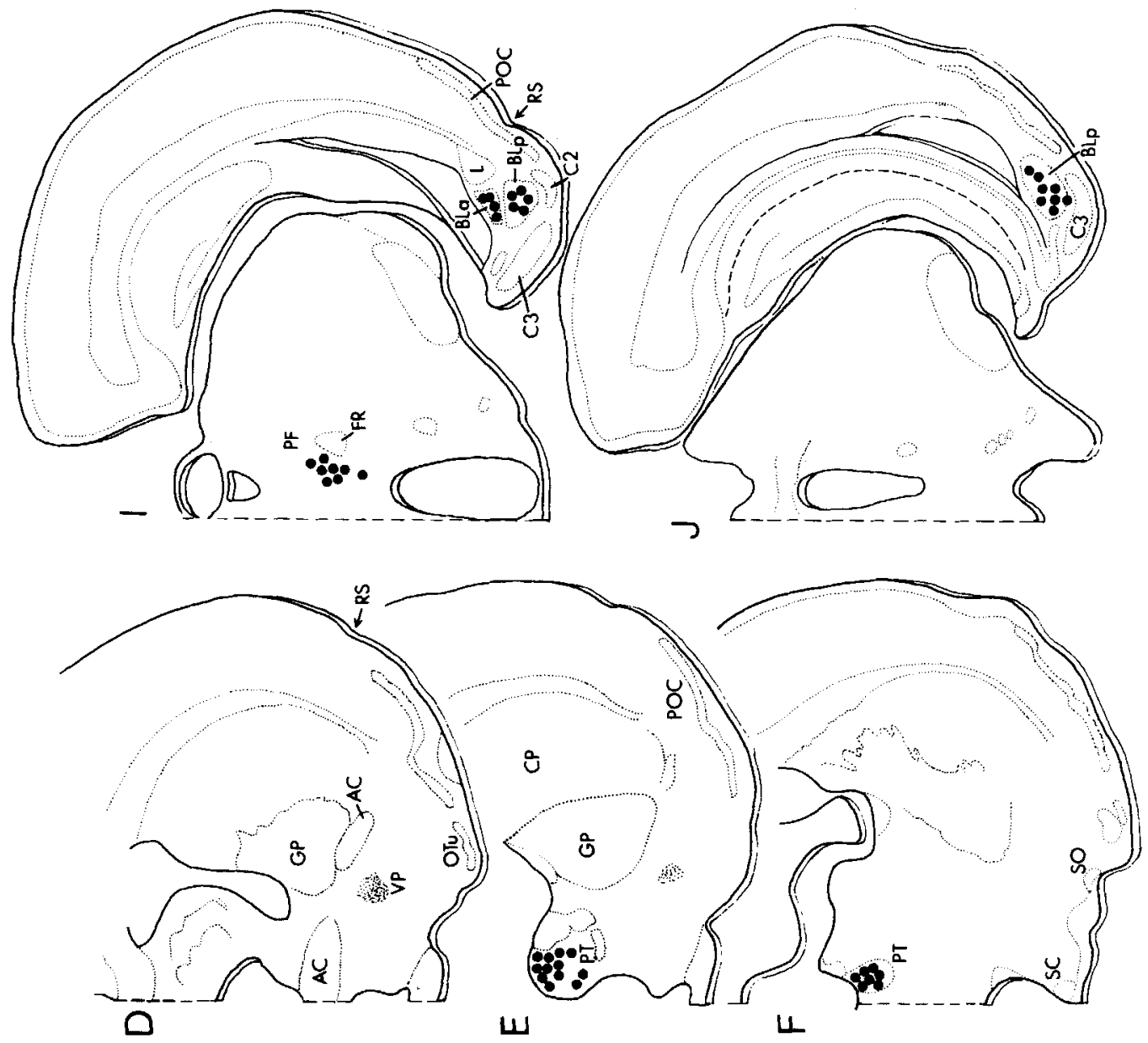

4 
cumbens in experiments 7711 or 7714 contained labeled cells in this experiment as well. Certain quantitative differences, however, were striking. The primary olfactory, posterior insular, and perirhinal cortices and entorhinal area $28 \mathrm{~L}$ all contained many more labeled neurons in this brain. Also, fewer labeled cells were present in the subiculum, and the majority of these were in the intermediate subiculum (Fig. 4K), in contrast to brains 7711 and 7714 in which both the dorsal and intermediate subiculum were heavily labeled by HRP applications in the rostral half of nucleus accumbens. In the amygdala, labeling was observed throughout the anterior division of the basolateral nucleus (Fig. 4F,G), but only in the rostral portion of the posterior division (Fig. 4H). Finally, the ventral tegmental area was poorly labeled, especially the caudal half (Fig. 4K,L).

Terminal fields were observed in this brain in the ventral pallidum (Figs. 4D; 11) and in the medial pars reticulata of the substantia nigra (Fig. 4K).

\section{Caudomedial nucleus accumbens}

The application site in experiment 7814 (Fig. 5) was only slightly caudal to that of experiment 7715 (Fig. 4), but was centered medial to the anterior limb of the anterior commissure, rather than lateral to it (Figs. $1 \mathrm{D} ; 5 \mathrm{~B}, \mathrm{C})$. The survival time in this case was 36 hours.

The pattern of labeling in the cortical areas of this brain differed remarkably from that observed in the three experiments described above. No labeled neurons were observed in the primary olfactory, posterior insular, or perirhinal cortices in this brain. Within the entorhinal cortex, labeling was present in area 28L' (Fig. 5L-N), but not in the more lateral area 28L, in contrast to brains having HRP applications in rostral, intermediate, or caudolateral nucleus accumbens (brains 7714, 7711 , and 7715), in which labeling was almost entirely confined to area 28L. Entorhinal area TR (of Haug, '76), medial to area $28 \mathrm{~L}$ ', also contained numerous well-labeled pyramidal cells in this brain (Fig. 5L). The intermediate subiculum contained many more labeled cells than were observed in brains having more lateral or rostral application sites, and the ventral subiculum was labeled as well (Fig. $5 \mathrm{~L}-\mathrm{N})$. No labeling was present in the dorsal subiculum or hippocampus.

Labeling in subcortical areas was similar to that observed after HRP applications in lat- eral or rostral parts of nucleus accumbens with the following exceptions: Within the thalamus, the paraventricular and central medial nuclei were not labeled; the basolateral nucleus of the amydala contained labeled neurons throughout its posterior division, but only caudally in its anterior division; the ventral tegmental area was poorly labeled; no labeling was found in the dorsal raphe. A terminal field was present in this brain only in the ventral pallidum (Fig. 5D,E).

\section{Contralateral labeling}

In each of the brains described above, a number of labeled neurons were observed in one or more contralateral cortical areas. These regions included the posterior insular, perirhinal, and entorhinal cortices. In experiments in which HRP was deposited in rostral, intermediate, and caudomedial nucleus accumbens (brains 7711, 7714, and 7814), this labeling was rather sparse, but in brain 7715 , in which the HRP application was centered in caudolateral nucleus accumbens and in which the ipsilateral cortical labeling was most extensive, these contralateral cortical areas contained a more substantial number of labeled cells. Occasional contralateral HRP-containing neurons were also present in several brains in the basolateral nucleus of the amygdala.

\section{Labeling in other areas}

In addition to the labeled areas discussed above, a few HRP-labeled cells were found in various brains in the dorsomedial, dorsal anterior, anteromedial, and rhomboid thalamic nuclei. Other lightly labeled areas included the magnocellular preoptic area and the periventricular central gray at the level of the diencephalic-mesencephalic junction. The results of control experiments described below suggest that cortical contamination probably produced the labeling observed in the thalamic nuclei. However, limited projections to nucleus accumbens from these nuclei and from the preoptic area and central gray could neither be proven nor ruled out.

Occasional labeled cells were also observed in many brains in the anterior cingulate and infralimbic cortices, adjacent to the needle or pipette tract. It seems likely that these neurons were labeled by direct uptake of HRP drawn into the cortex along the needle or pipette tract.

Finally, in each brain described above, large, densely labeled neurons were present 
in the ventral pallidum, scattered within the terminal field there. Because these cells were so heavily packed with reaction product, it was felt that their axons had been damaged by the micropipette, allowing HRP to enter the cells by diffusion. While it is possible that these cells are efferent to nucleus accumbens, it seems more likely that they project through accumbens to more rostral regions. Cells in this area, for example, are known to project to the frontal cortex (Heimer, '78), anterior olfactory nucleus (Swanson, '76; Haberly and Price, '78), and olfactory bulb (de Olmos et al., '78).

\section{Control experiments}

In the experiments described above, only trace amounts of HRP were deposited in the frontal cortex overlying nucleus accumbens as the pipette was withdrawn from the brain. Nevertheless, because many of the labeled areas in these experiments are known to project to the frontal cortex (Jones and Leavitt, '74; Beckstead, '76; Krettek and Price, '77b), in several brains, applications of HRP were made in the cortex dorsal to accumbens in order to evaluate the possibility that some labeling resulted from cortical contamination. Although these applications were relatively small, the amount of HRP deposited in the frontal cortex in these brains was much greater than that resulting from applications of HRP in nucleus accumbens. Areas containing a significant number of labeled cells in both the experimental brains and the control brains included the paraventricular thalamic nucleus and the dorsal raphe. However, the quantity of labeled neurons in these areas in the control brains was less than in the experimental brains, despite the much greater amounts of HRP deposited in the cortex in the control experiments. These control experiments, then, demonstrated that the labeling observed in the experimental brains was not due to retrograde transport from HRP-contaminated cortex.

Control HRP applications were also made in the anterior olfactory nucleus in several brains, as was mentioned above.

\section{DISCUSSION}

\section{Technical considerations}

The HRP technique has certain qualifications which must be considered when interpreting data derived by this method. In some cases, the method of retrograde transport of
HRP has failed to demonstrate connections which have been shown to exist by other techniques (Nauta et al., '74). Therefore, it is possible that afferents to nucleus accumbens might exist in addition to those demonstrated here. The use of orthograde transport of HRP to demonstrate efferent connections must be presumed to have the same possible limitation. In addition, it was found here that the amount of HRP required in an area for orthograde transport to occur is greater than that necessary for retrograde transport. Consequently, it may not always be possible to restrict an HRP application to the area under investigation and at the same time deposit enough HRP to insure successful orthograde transport.

Fibers-of-passage may also complicate the interpretation of HRP experiments in some cases, since damaged axons may take up HRP and transport it in a retrograde direction (Halperin and LaVail, '75), and Hedreen and McGrath ('78) have shown that HRP may enter undamaged axons and label terminals and perikarya by intraaxonal diffusion. However, this labeling by diffusion is not likely to have been a factor in the experiments described above, since it disappears in most axons within 24 hours. With the exception of the retrograde labeling observed in the ventral pallidum, it is also unlikely that transport by damaged fibers-of-passage was a significant problem. Since the needle used for hydraulic injections caused considerably more tissue damage than the micropipettes used for iontophoresis, the patterns of cell labeling observed with the two techniques should have been different if significant retrograde transport was occurring along damaged fibers-ofpassage. However, differences were not noted.

Perhaps the most important factor to consider when using the HRP technique is the method of defining the region of an application site from which transport can occur. As mentioned above, the size of this region is probably smaller for orthograde than for retrograde transport, and undoubtedly differs somewhat for each group of afferent and efferent neurons of the area under investigation (de Olmos, '77). In addition, the apparent size of an application site is influenced by the survival time and by the particular reaction used to visualize the HRP. In the present study, it was assumed that transport occurred only from regions (1) and (2) of the application sites, since the concentration of HRP in region (3) was very low. This was appropriate in the case 
of retrograde transport, since the results obtained when all three regions of the application site were within nucleus accumbens were similar to those obtained when only regions (1) and (2) were within the nucleus. However, this assumption may have overestimated the area from which orthograde transport could occur, as will be seen below.

\section{Afferent connections of nucleus accumbens}

Previously unreported sources of afferents to nucleus accumbens identified here include the posterior agranular insular, perirhinal, and entorhinal cortices. These areas, as well as the basolateral amygdala, may project to both the ipsilateral and contralateral nucleus accumbens. The possibility of contralateral cortical afferents to nucleus accumbens is consistent with the findings of van Alphen ('69).

Although the afferent connections of nucleus accumbens and their topographical organization have never been systematically studied prior to this investigation in the hamster, a number of the projections to nucleus accumbens reported here have been noted in anterograde tracing experiments in other species. Cowan et al. ('65) observed degeneration in nucleus accumbens after placing lesions in the amygdala, and Krettek and Price ('78a), using the autoradiographic technique, demonstrated projections to nucleus accumbens of the rat and cat from the basolateral and basomedial amygdaloid nuclei, and from the amygdalohippocampal area. In the present study, amygdaloid efferents were found to nucleus accumbens from the basolateral nucleus only. The basomedial nucleus of the hamster is very poorly developed, and it is likely that the absence of basomedial afferents reflects this lack of development. While no labeling was observed in the present study in the amygdalohippocampal area, the adjacent subiculum and entorhinal area TR were shown to be efferent to nucleus accumbens. It is possible, therefore, that in the experiments of Krettek and Price, diffusion of tritiated amino acid into these areas occurred from injection sites in the amygdala.

In the present study in the hamster, the entire subiculum was shown to project to nucleus accumbens. In contrast, Swanson and Cowan (77) found only the ventral portion of the subiculum to be efferent to nucleus accumbens in the rat. This difference apparently represents an interspecific variation.

In the rat, nucleus accumbens is known to receive dopaminergic afferents from the ven- tral tegmental area, substantia nigra, and cell group A8 of Dählstrom and Fuxe ('64) (Ungerstedt, '71; Fallon and Moore, '78; Nauta et

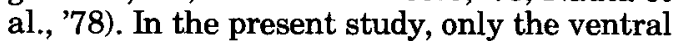
tegmental area was found to project to nucleus accumbens. Fallon and Moore ('78) and Björklund and Lindvall (78) have described the dopaminergic cells of the ventral midbrain as a single nuclear group whose neurons project topographically upon the forebrain. The absence of projections to nucleus accumbens from the substantia nigra or area A8 in the hamster is consistent with this concept, since it may simply reflect differences in the topographic organization of the dopaminergic "nucleus" in these two species.

Other areas reported to project to nucleus accumbens on the basis of anterograde experiments, which were found to be sources of afferents to accumbens in this study as well, include the olfactory cortex (de Olmos and Ingram, '72; Heimer and Wilson, '75), hippocampus (Fox, '43; Raisman et al., '66; de Olmos and Ingram, '72; Siegel and Tassoni, '71a; Siegel et al., '75; Heimer and Wilson, '75), thalamic nuclei parataenialis, paraventricularis, and reuniens (Cowan and Powell, '55; Powell and Cowan, '56; Swanson and Cowan, '75; Herkenham, '78), and the dorsal raphe (Bobillier et al., '76).

Several previously reported projections to nucleus accumbens were not observed in this study. The prelimbic, anterior cingulate, and rostral sulcal cortices were reported to be efferent to the nucleus accumbens of the rat by Beckstead ('79). In the present study, no labeling was observed in the rostral sulcal cortex following applications of HRP in accumbens. Occasional HRP-labeled cells were present in the anterior cingulate and infralimbic cortices in most brains, adjacent to the needle or pipette tract. While sparse projections to nucleus accumbens from these latter cortical areas in the hamster cannot be ruled out, it seems likely that this labeling was due to the uptake of HRP drawn into the cortex along the needle or pipette tract.

Siegel and Tassoni ('71b) have proposed that the septum projects to nucleus accumbens, in contrast to the findings reported here. It is likely that the degenerating axons observed in nucleus accumbens by these investigators were hippocampal, entorhinal, or subicular fibers passing to nucleus accumbens in the fornix, which were damaged by the lesions placed in the septum. This interpretation is supported by the autoradiographic experiments of Swanson and Cowan ('79). 
Degeneration in nucleus accumbens following placement of lesions in the anterior medial thalamic nucleus of the rat was described by Leonard ('69). Such a projection was not conclusively demonstrated in the present study in the hamster. It is possible that Leonard's lesions damaged fibers passing to accumbens from thalamic nuclei adjacent to anteromedialis, such as paraventricularis, parataenialis, or centromedialis.

Finally, Fallon et al. ('78), using the HRP method, have suggested that nucleus accumbens receives afferents from the islands of Calleja. This projection was not observed in the present study, even though the TMB technique employed here is more sensitive than the DAB technique used by Fallon et al. It seems likely that the large injections of HRP employed by Fallon et al. allowed HRP to reach the island cells by diffusion, rather than by retrograde transport.

\section{Efferent connections of nucleus accumbens}

In the present study, a dense terminal field was observed in the ventral pallidum in all of the brains, in agreement with findings in the rat by Heimer and Wilson ('75), Domesick et al. ('76), and Nauta et al. ('78).

Efferents from nucleus accumbens to the substantia nigra have also been described by a number of investigators (Swanson and Cowan, '75; Conrad and Pfaff, '76; Domesick et al., '76). In the present study, faint terminal fields were present in the pars reticulata of the substantia nigra only in brains 7714 and 7715 . Since these terminal fields were quite faint, it is possible that inadvertent slight differences in processing prevented similar fields from being demonstrated in brains 7711 and 7814 . Less-rapid dehydration of the brain sections in ethanol, for example, could render a faint terminal field undetectable, as alcohol causes fading of the HRP reaction product, especially product contained in axon terminals.

In contrast to the findings described here, Nauta et al. ('78), using the autoradiographic technique in the rat, reported a projection from the nucleus accumbens to the ventral tegmental area, and Phillipson ('79) observed labeling in nucleus accumbens after HRP applications in the rat ventral tegmental area. However, ventral tegmental area efferents

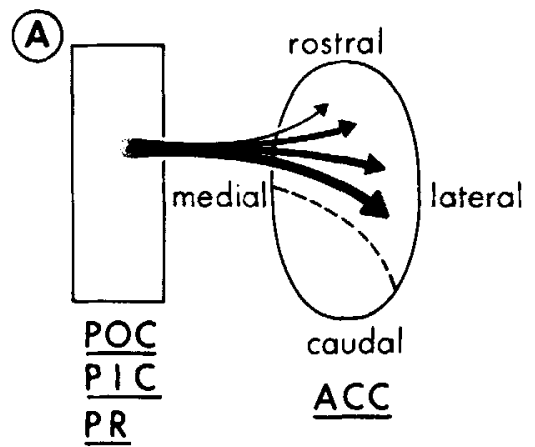

(B)
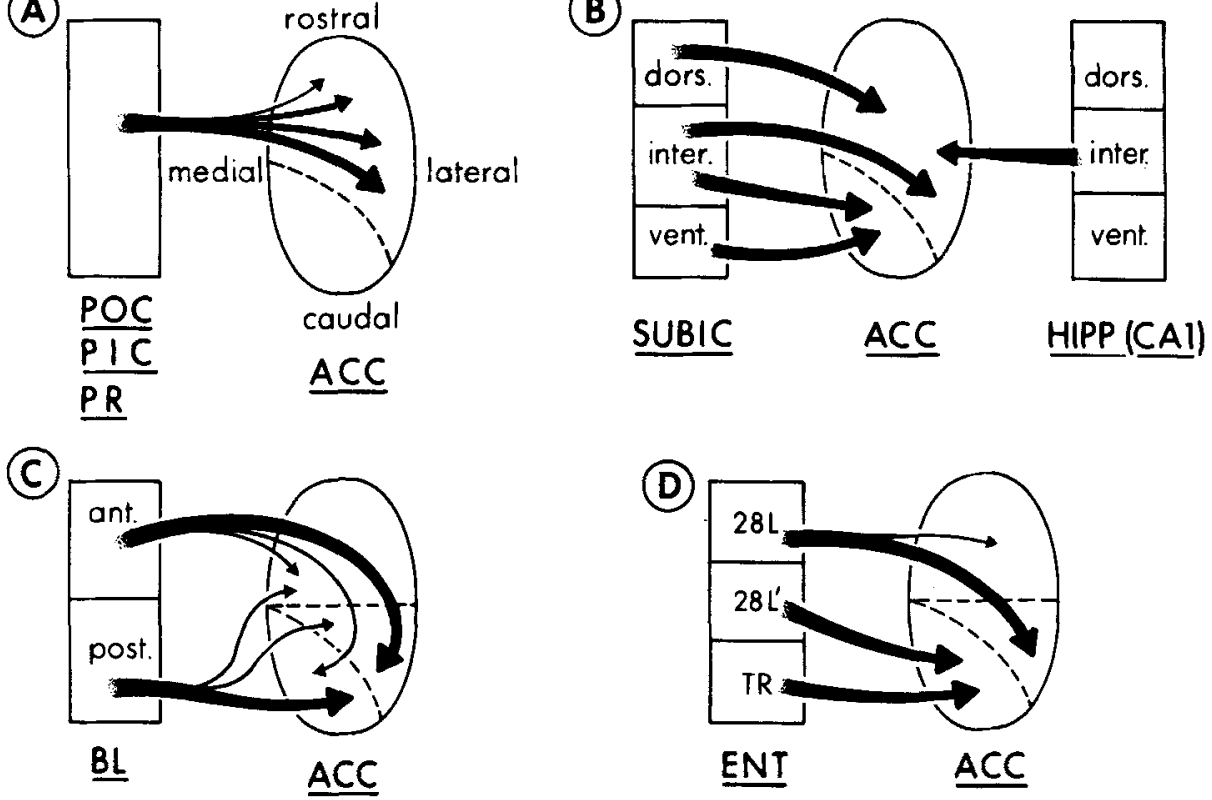

Fig. 6. A series of schematic diagrams illustrating the topographical organization of the projections to accumbens from (A) the primary olfactory, posterior insular, and perirhinal cortices; (B) the subiculum (SUBIC) and hippocampus (HIPP); (C) the anterior and posterior division of the basolateral amygdaloid nucleus (BL); and (D) entorhinal (ENT) areas 28L, 28L', and TR. Within a given projection system, heavy projections are indicated by wide arrows, lighter projections by thinner arrows. 


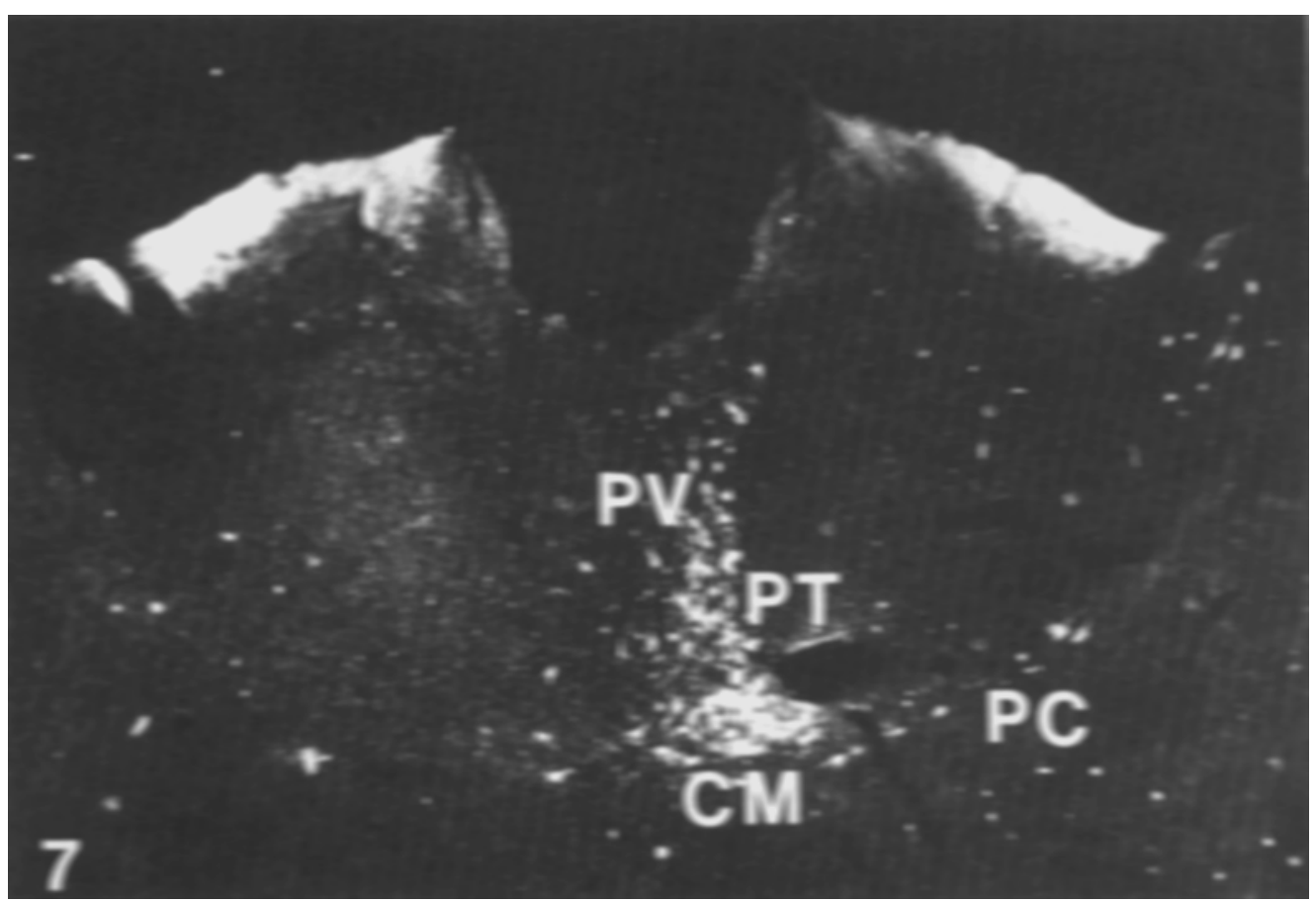

Fig. 7. Darkfield photomicrograph of a coronal section through the thalamus of brain 7714, corresponding to Figure 4G, showing HRP-labeled neurons in the paraventricular, parataenial, central medial, and paracentral nuclei.

from nucleus accumbens were not noted in autoradiographic studies in the rat by Swanson and Cowan (75) and Conrad and Pfaff ('76).

Numerous other efferent connections of nucleus accumbens have been described in studies employing autoradiographic or degeneration techniques, including projections to the globus pallidus, septum, hypothalamus, preoptic area, hippocampus, frontal cortex, olfactory tubercle, and thalamus (Swanson and Cowan, '75; Conrad and Pfaff, '76; Williams et al., '77; Fallon et al., '78; Herkenham, '78; Nauta et al., '78). Some of the limitations of HRP technique discussed earlier may have prevented the demonstration of these connections in the present study. In particular, the method used here for defining the limits of an effective HRP application might have overestimated the size of the area from which orthograde transport could occur; perhaps larger HRP applications would have labeled additional terminal fields. On the other hand, the use of autoradiographic and degeneration tech- niques has clearly yielded no concensus on the efferent projections of nucleus accumbens. The studies cited above are in agreement only on projections to the substantia nigra and pallidum, the two areas also identified here; in other respects they often differ markedly. It is likely, therefore, that at least some of the projections described in those reports were incorrectly ascribed to accumbens.

\section{Topographical organization of the connections of nucleus accumbens}

This study demonstrates that a number of the afferent projections to nucleus accumbens are topographically organized. As diagrammed in Figure 6A, the primary olfactory, posterior agranular insular, and perirhinal cortical areas project most heavily to caudolateral nucleus accumbens. Labeling in these cortices was very light when HRP was deposited in the rostral pole of the nucleus (brain 7714; Fig. 2), but increased progressively in brains having HRP applications in intermediate (brain 7711; Fig. 3) or caudolateral 


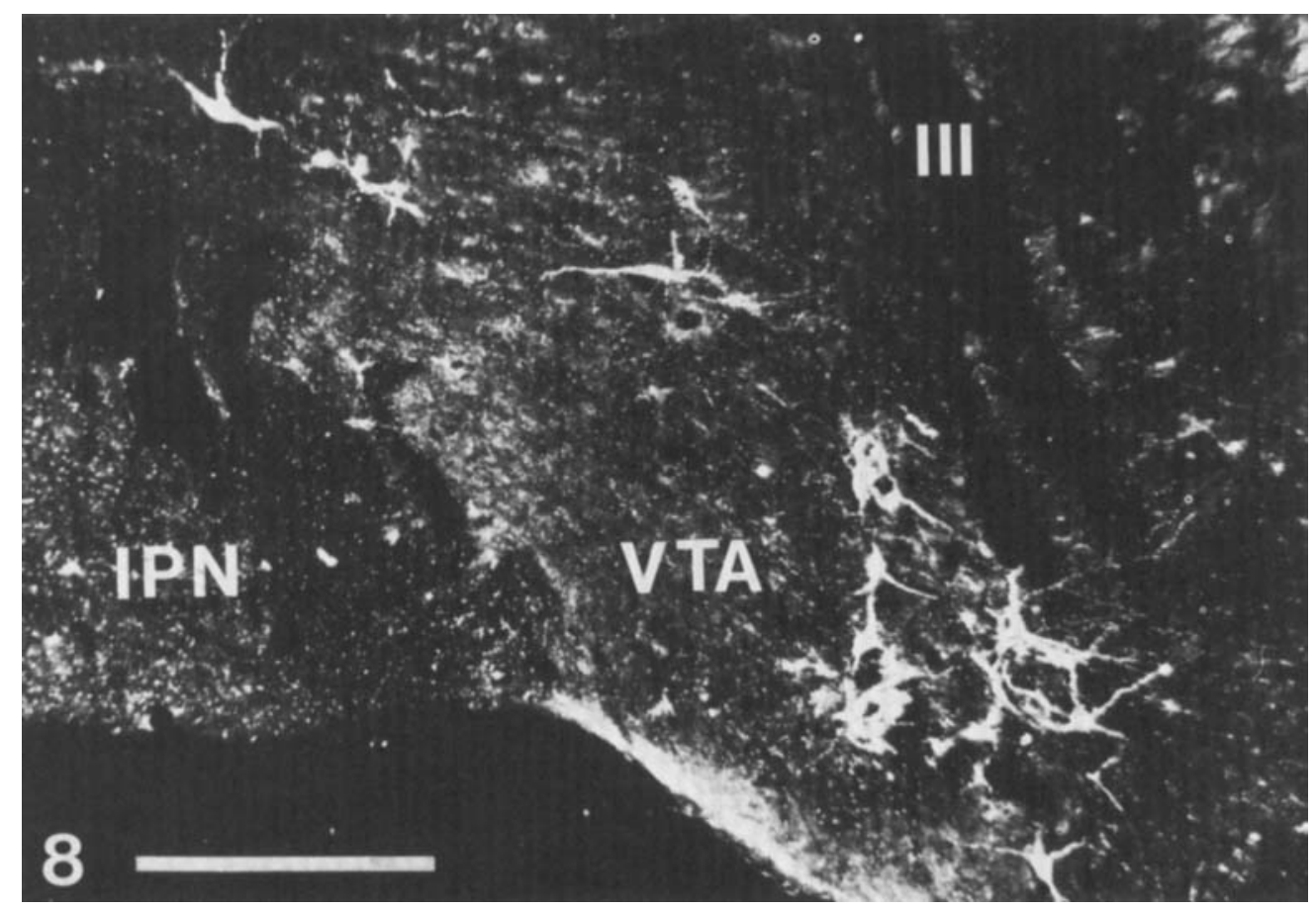

Fig. 8. Darkfield photomicrograph of a coronal section through the midbrain of brain 7714, corresponding to Figure $4 \mathrm{~L}$, showing HRP-containing neurons in the ventral tegmental area. III, oculomotor nerve. Calibration bar, $200 \mu \mathrm{m}$ for this figure; $500 \mu \mathrm{m}$ for Figures 7 and 9.

(brain 7715; Fig. 4) nucleus accumbens. No labeling was observed in these cortical areas after an HRP application in caudomedial nucleus accumbens (brain 7814; Fig. 5). The projections from these cortices are topographic only with respect to their termination within nucleus accumbens. All parts of this nucleus receiving fibers from the insular and perirhinal regions do so from neurons distributed throughout these areas, and though the olfactory cortical projection originates mainly in a restricted region of the olfactory cortex between the levels of the rostral and middle amygdala (Fig. 4F-H), in each brain in which the olfactory cortex was labeled, HRP-containing neurons were present throughout this region.

In contrast, the efferents to accumbens from the subiculum and entorhinal cortex display point-to-point specificity (Fig. 6B,D). An application of HRP in rostral nucleus accumbens (brain 7714; Fig. 2) labeled cells in the rostral dorsal subiculum and rostral intermediate subiculum, while caudal areas of the dorsal and intermediate subiculum were labeled by HRP deposited in the intermediate portion of the nucleus (brain 7711; Fig. 3). After an HRP application in caudolateral nucleus accumbens (brain 7715; Fig. 4), many HRP-containing cells were present in the intermediate subiculum, but only a few were observed in the dorsal subiculum. The ventral subiculum and caudal-most intermediate subiculum were labeled only after a caudomedial HRP application in nucleus accumbens (brain 7814; Fig. $5 \mathrm{~K}-\mathrm{M})$. The entorhinal projection to nucleus accumbens originates in lateral areas $28 \mathrm{~L}$, $28 \mathrm{~L}^{\prime}$, and TR, and is directed almost exclusively at the caudal pole of the nucleus (Fig. 6D). Areas $28 \mathrm{~L}^{\prime}$ and TR project only to the caudomedial part of nucleus accumbens (Fig. 5I-K; brain 7814) while axons from area $28 \mathrm{~L}$ terminate in the caudolateral portion of the nucleus (Fig. 4F,G; experiment 7715). 


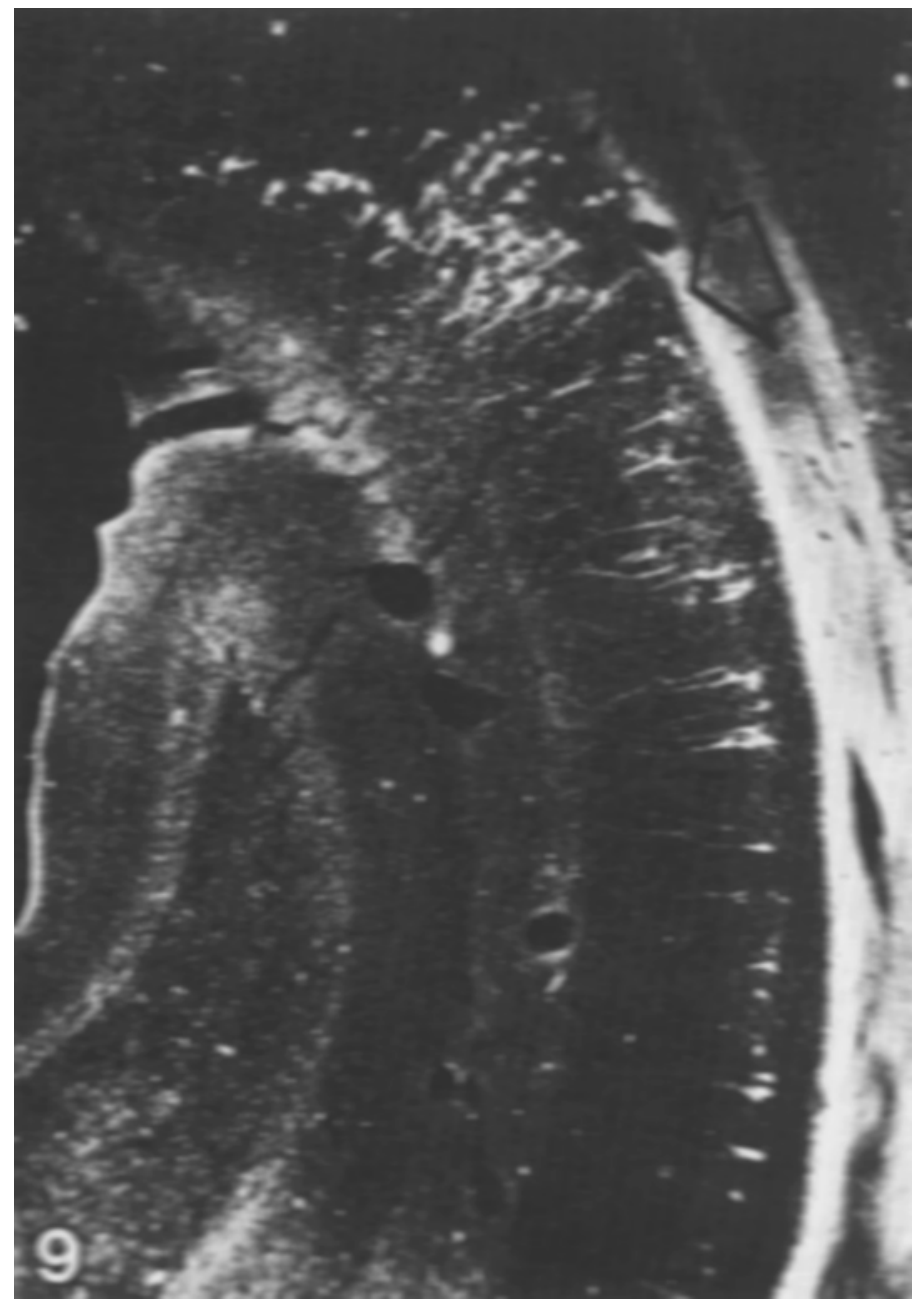

Fig. 9. Darkfield photomicrograph of a coronal section of brain 7714, corresponding to Figure 4K, showing HRP-labeled pyramidal cells in the dorsal subiculum (above arrow) and in hippocampal field CA1 (below arrow).

The intermediate portion of hippocampal field CA1 was found to be efferent to rostral nucleus accumbens in the present study (Fig. 6B). Heimer and Wilson ( 75, p. 181) have diagrammed a hippocampal projection to cau- dal as well as rostral nucleus accumbens. It is possible that the degeneration they observed in the caudal area following ablation of the rat hippocampus was due to the destruction of subicular or entorhinal fibers-of-passage pro-

Fig. 10. Darkfield photomicrograph of a coronal brain section through the nucleus accumbens, showing a large iontophoretic HRP application centered in accumbens and extending into surrounding regions. 1, 2, and 3, zones of application site, as described in the text.

Fig. 11. Darkfield photomicrograph of a coronal section of brain 7711 corresponding to Figure 2E, pointing out a dense terminal field in the ventral pallidum. Calibration bar, $500 \mu \mathrm{m}$ for Figures 10 and 11. 

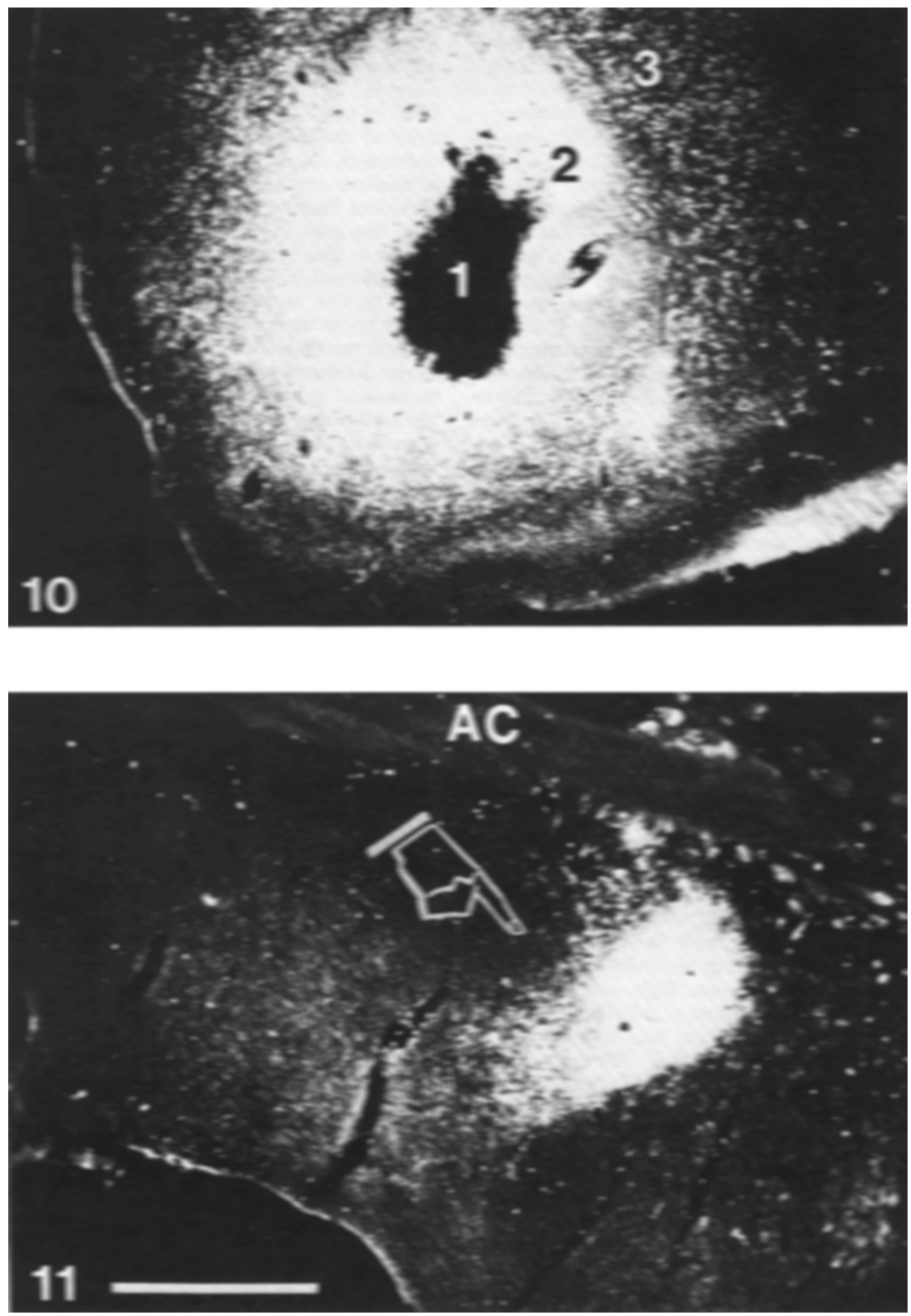
jecting to the caudal part of the nucleus through the fimbria.

Figure 6C shows that the afferents to nucleus accumbens from the basolateral nucleus of the amygdala terminate most extensively in the caudal portion of accumbens; labeling in this nucleus was sparse when HRP was deposited in the rostral pole of accumbens (brain 7714; Fig. 2), but was considerably more extensive in brains having more caudal application sites (experiments 7711, 7715, 7814; Figs. 3-5). Furthermore, labeling in the anterior division of the basolateral nucleus was heaviest when HRP was deposited in caudolateral nucleus accumbens (brain 7715; Fig. 4), while HRP applications in caudomedial accumbens labeled the posterior division most heavily (brain 7814; Fig. 5). These latter results confirm the findings of Krettek and Price ('78a) in the rat and cat.

Intralaminar thalamic efferents to accumbens arise from the rostral portions of the central medial and paracentral nuclei and from rostral parafascicular neurons ventromedial to the fasciculus retroflexus. The origin of these projections in restricted portions of the intralaminar nuclei is in contrast to the midline thalamic projections to nucleus accumbens, which arise throughout their respective nuclei. The pattern of termination of the intralaminar and midline thalamic projections in nucleus accumbens is such that all parts of nucleus accumbens are innervated by the parafascicular, parataenial, and reuniens nuclei, while the central medial and paraventricular nuclei project to all but the caudomedial portion of the nucleus (Fig. 5; brain 7814).

The projection to nucleus accumbens from the ventral tegmental area displays a rostrocaudal topography, such that the ventral tegmental area efferents terminate most heavily in rostral accumbens. This can be seen by examining the amount of labeling in the ventral tegmental area in experiments having successively more caudal HRP application sites (brains 7714, 7711, 7715, and 7814; Figs. $2-5)$. In contrast to the findings of Fallon and Moore ('78) in the rat, this projection was not found to display point-to-point topography, and a medial-lateral component was not noted in the topography of these fibers.

The data obtained here with HRP on the efferents of nucleus accumbens support the findings most consistently reported with autoradiographic and degeneration techniques, but do not provide evidence for a topographical organization of these projections. While the substantia nigra was found to receive efferents from the rostral and caudolateral portions of nucleus accumbens, a terminal field was not observed in the nigra after an application of HRP in the caudomedial or intermediate portions of the nucleus. However, the apparent absence of nigral projections from these parts of nucleus accumbens may have been due to technical factors as discussed above. Therefore, the possibility of topographical organization in this projection cannot be evaluated. The efferent projection from nucleus accumbens to the ventral pallidum was also not found to be topographically organized in the present study, in contrast to the finding in the rat of Swanson and Cowan ('75) that medial portions of the nucleus project medially within the pallidum, and efferents from lateral parts of nucleus accumbens terminate laterally.

The experimental results described in this paper substantiate the classification of nucleus accumbens as a striatal structure and provide experimental evidence supporting the concept of the ventral striatum. Since experiments described in the second paper in this series on the connections of the olfactory tubercle (Newman and Winans, this issue) provide additional information concerning the organization and possible function of the ventral striatal system, the evidence for the above statements will be discussed in that paper, together with the results of the olfactory tubercle experiments.

\section{ACKNOWLEDGMENTS}

We gratefully acknowledge the fine artistic contributions of our colleague William L. Brudon, and the thoughtful criticism of the manuscript by Dr. Lennart Heimer.

This research was supported in part by NIMH grant MH29375. Dr. Newman was the recipient of a Student Research Fellowship from the University of Michigan Medical School.

\section{LITERATURE CITED}

Beckstead, R.M. (1976) Convergent thalamic and mesencephalic projections to the anterior medial cortex in the rat. J. Comp. Neurol., 166:403-416.

Beckstead, R.M. (1979) An autoradiographic examination of corticocortical and subcortical projections of the mediodorsal projection (prefrontal) cortex in the rat. J. Comp. Neurol., 184:43-62.

Björklund, A, and O. Lindvall (1978) The mesotelencephalic dopamine neuron system: A review of its anatomy. In: Limbic Mechanisms: The Continuing Evolution of the Limbic System Concept. K. E. Livingston and O. Hornykiewicz, eds. Plenum Press, New York, pp. 307-332. 307-332. 
Bobillier, P., S. Seguin, F. Petitjean, D. Salvert, M. Touret, and $M$. Jouvent (1976) The raphe nuclei of the brainstem: A topographical atlas of their efferent projections as revealed by autoradiography. Brain Res., 113:449-486.

Colman, D., F. Scalia, and E. Cabrales (1976) Light and electron microscopic observations on the anterograde transport of horseradish peroxidase in the optic pathway in the mouse and rat. Brain Res., 102:156-163.

Conrad, L.C.A., and D.W. Pfaff (1976) Autoradiographic tracing of nucleus accumbens efferents in the rat. Brain Res., 113:589-596.

Cowan, W.M., and T.P.S. Powell (1955) The projections of the midline and intralaminar nuclei of the thalamus of the rabbit. J. Neurol. Neurosurg. Psychiat., 18:266-279.

Cowan, W.M., G. Raisman, and T.P.S. Powell (1965) The connexions of the amygdala. J. Neurol. Neurosurg. Psychiat., 28:137-151.

Dählstrom, A., and K. Fuxe (1964) Evidence for the existence of monamine containing neurons in the central nervous system. I. Demonstration of monamines in the cell bodies of brainstem neurons. Acta Physiol. Scand., 62 (Suppl. 232):1-55.

de Olmos, J.S. (1977) An improved HRP method for the study of central nervous connections. Exp. Brain Res., 29:541-551.

de Olmos, J., H. Hardy, and L. Heimer (1978) The afferent connections of the main and accessory olfactory bulb formations in the rat: An experimental HRP-study. J. Comp. Neurol., 181:213-244.

de Olmos, J.S., and W.R. Ingram (1972) The projection field of the stria terminalis in the rat brain. An experimental study. J. Comp. Neurol., 146:303-334.

Dill, R.E., and E. Costa (1977) Behavioral dissociation of the enkephalinergic system of accumbens and the nucleus caudatus. Neuropharmacology, 16:323-326.

Domesick, V.B., G.P. Smith, and W.J.H. Nauta (1976) Contrasting projections of the nucleus accumbens and caudatoputamen in the rat. Abstract, Cajal Club Proceedings.

Fallon, J.H., and R.Y. Moore (1978) Catecholamine innervation of the basal forebrain. IV. Topography of the dopamine projection to the basal forebrain and neastriatum. J. Comp. Neurol., 180:545-580.

Fallon, J.H., JN. Riley, J.C. Sipe, and R.Y. Moore (1978) The islands of Calleja: Organization and connections. J. Comp. Neurol., 181:375-396.

Fox, C.A. (1943) The stria terminalis, longitudinal association bundle, and precommissural fornix fibers in the cat. J. Comp. Neurol., 79:277-295.

Graham, R.C., and M.J. Karnovsky (1966) The early stages of absorption of injected horseradish peroxidase in the proximal tubules of mouse kidney: Ultrastructural cytochemistry by a new technique. J. Histochem. Cytochem., 14:291-303.

Guillery, R.W. (1959) Afferent fibers to the dorso-medial thalamic nucleus in the cat. J. Anat., 93:403-419.

Gurdjian, E.S. (1927) The diencephalon of the albino rat. J. Comp. Neurol., 43:1-114.

Gurdjian, E.S. (1928) The corpus striatum of the rat. J. Comp. Neurol., 45:249-281.

Haberly, L.B., and J.L. Price (1978) Association and commissural fiber systems of the olfactory cortex of the rat. I. Systems originating in the piriform cortex and adjacent areas. J. Comp. Neurol., 78:711-740.

Halperin, J.J., and J.H. LaVail (1975) A study of the dynamics of retrograde transport and accumulation of horseradish peroxidase in injured neurons. Brain Res., 100:253-270.
Hardy, H., and L. Heimer (1977) A safer and more sensitive substitute for diaminobenzidine in the light microscopic demonstration of retrograde and anterograde axonal transport of HRP. Neurosci. Lett., 5:235-240.

Haug, F.-M.S. (1976) Sulphide silver pattern and cytoarchitectonics of parahippocampal areas in the rat. Adv. Anat. Embryol. Cell Biol., 52:1-73.

Hedreen, J.C., and S. McGrath (1978) Observations on labeling of neuronal cell bodies, axons, and terminals after injection of horseradish peroxidase into rat brain. J. Comp. Neurol., 176:225-246.

Heimer, L. (1978) The olfactory cortex and the ventral striatum. In: Limbic Mechanisms: The Continuing Evolution of the Limbic System Concept. K. E. Livingston and O. Hornykiewicz, eds. Plenum Press, New York, pp. 95-187.

Heimer, L., and R.D. Wilson (1975) The subcortical projections of the allocortex: Similarities in the neural connections of the hippocampus, the piriform cortex, and the neocortex. In: Golgi Centennial Symposium: Perspectives in Neurobiology. M. Santini, ed. Raven, New York, pp. 177-193.

Herkenham, M. (1978) The connections of nucleus reuniens thalami: Evidence for a direct thalamo-hippocampal pathway in the rat. J. Comp. Neurol., 177:589-610.

Jones, E.G., and R.Y. Leavitt (1974) Retrograde axonal transport and the demonstration of nonspecific projections to the cerebral cortex and striatum from thalamic intralaminar nuclei in the rat, cat, and monkey. J. Comp. Neurol., 154:349-378.

Knook, H.L. (1966) The Fiber Connections of the Forebrain. F.A. Davis, Philadelphia, p. 148.

Koikegami, H., Y. Hirata, and J. Ogrema (1967) Studies on the paralimbic brain structures and some experiments on the nucleus accumbens. Folia Psychiat. Neurol. Jpn., 21:151-180.

Krettek, J.E., and J.L. Price (1977a) The cortical projections of the mediodorsal nucleus and adjacent thalamic nuclei in the rat. J. Comp. Neurol., 171:157-192.

Krettek, J.E., and J.L. Price (1977b) Projections from the amygdaloid complex to the cerebral cortex and thalamus in the rat and cat. J. Comp. Neurol., 172:687-722.

Krettek, J.E., and J.L. Price (1978a) Amygdaloid projections to subcortical structures within the basal forebrain and brainstem in the rat and cat. J. Comp. Neurol., 178:225-254.

Krettek, J.E., and J.L. Price (1978b) A description of the amygdaloid complex in the rat and cat with observations on intraamygdaloid axonal connections. J. Comp. Neurol., 178: 255-280.

Lauer, E.W. (1945) The nuclear pattern and fiber connections of certain basal telencephalic centers in the macaque. J. Comp. Neurol., 82:215-245.

Leonard, C.M. (1969) The prefrontal cortex of the rat. I. Cortical projections of the mediodorsal nucleus. II. Efferent connections. Brain Res., 12:321-343.

Nauta, H.J.W., M.B. Pritz, and R.J. Lasek (1974) Afferents to the rat caudatoputamen studied with horseradish peroxidase. An evaluation of a retrograde neuroanatomical research method. Brain Res., 67:219-238.

Nauta, W.J.H., G.P. Smith, R.L.M. Faull, and V.B. Domesick (1978) Efferent connections and nigral afferents of the nucleus accumbens in the rat. Neuroscience 3:385-401.

Newman, R., and S.S. Winans (1980) An experimental study of the ventral striatum of the golden hamster. Il. The neuronal connections of the olfactory tubercle. (this issue). 
Phillipson, O.T. (1979) Afferent projections to the ventral tegmental area of Tsai and interfascicular nucleus: A horseradish peroxidase study in the rat. J. Comp. Neurol., 187:117-143.

Pijenburg, A.J.J., G.N. Woodruff, and J.M. van Rossum (1973) Ergometrine induced locomotor activity following intracerebral injection into the nucleus accumbens. Brain Res., 59:289-302.

Powell, T.P.S., and W.M. Cowan (1956) A study of thalamo-striate relations in the monkey. Brain, 79:364-390.

Raisman, G., W.M. Cowan, and T.P.S. Powell (1966) An experimental analysis of the efferent projections of the hippocampus. Brain, 89:83-108.

Rioch, D.M. (1931) Certain myelinated fiber connections of the diencephalon of the dog, cat, and aevisa. J. Comp. Neurol., 53:319-388.

Siegel, A., S. Ohgami, and H. Edinger (1975) Projections of the hippocampus to the septal area in the squirrel monkey. Brain Res., 99:247-260.

Siegel, A., and J.P. Tassoni (1971a) Differential efferent projections from the ventral and dorsal hippocampus of the cat. Brain Behav. Evol., 4:185-200.

Siegel, A., and J.P. Tassoni (1971b) Differential efferent projections of the lateral and medial septal nuclei to the hippocampus in the cat. Brain Behav. Evol., 4:201-219.

Smith, M.O., and R. Holland (1975) Effects of lesions of the nucleus accumbens on lactation and postpartum behavior. Physiol. Psychol., 3:331-350.
Smith, O.C. (1930) The corpus striatum, amygdala, and stria terminalis of Tamandua tetradactyla. J. Comp. Neurol., 51:65-127.

Swanson, L.W. (1976) An autoradiographic study of the efferent connections of the preoptic area in the rat. $J$. Comp. Neurol., 167:227-256.

Swanson, L.W., and W.M. Cowan (1975) A note on the connections and development of the nucleus accumbens. Brain Res., 92:324-330.

Swanson, L.W., and W.M. Cowan (1977) An autoradiographic study of the organization of the efferent connections of the hippocampal formation in the rat. J. Comp. Neurol., 172:49-84.

Swanson, L.W., and W.M. Cowan (1979) The connections of the septal region in the rat. J. Comp. Neurol., 186:621-655.

Ungerstedt, U. (1971) Stereotaxic mapping of the monamine pathways in rat brain. Acta Physiol. Scand. 197 (Suppl. 367):1-48.

van Alphen, H.A.M. (1969) The anterior commissure of the rabbit. Acta Anat. (Basel), 74 (Suppl. 57):1-112.

Williams, D.J., A.R. Crossman, and P. Slater (1977) The efferent projections of the nucleus accumbens in the rat. Brain Res., 130:217-227. 